\title{
Clinical value of microRNA-198-5p downregulation in lung adenocarcinoma and its potential pathways
}

\author{
SHI-SHUO WANG ${ }^{1 *}$, YE-YING FANG ${ }^{2 *}$, JIA-CHENG HUANG ${ }^{1}$, YUE-YA LIANG $^{1}$, \\ YI-NAN GUO $^{1}$, LIN-JIANG PAN ${ }^{2}$ and GANG CHEN ${ }^{1}$ \\ ${ }^{1}$ Department of Pathology, The First Affiliated Hospital of Guangxi Medical University; \\ ${ }^{2}$ Department of Radiation Oncology, The First Affiliated Hospital of Guangxi Medical University, \\ Radiation Oncology Clinical Medical Research Center of Guangxi, Nanning, \\ Guangxi Zhuang Autonomous Region 530021, P.R. China
}

Received December 1, 2018; Accepted June 13, 2019

DOI: $10.3892 / 01.2019 .10610$

\begin{abstract}
Lung adenocarcinoma (LUAD), the main subtype of non-small cell lung cancer, is known to be regulated by various microRNAs (miRs/miRNAs); however, the role of miR-198-5p in LUAD has not been clarified. In the present study, the clinical value of miR-198-5p in LUAD and its potential molecular mechanism was evaluated. miR-198-5p expression was examined by reverse transcription-quantitative
\end{abstract}

Correspondence to: Professor Lin-Jiang Pan, Department of Radiation Oncology, The First Affiliated Hospital of Guangxi Medical University, Radiation Oncology Clinical Medical Research Center of Guangxi, 6 Shuangyong Road, Nanning, Guangxi Zhuang Autonomous Region 530021, P.R. China

E-mail:67666795@qq.com

Professor Gang Chen, Department of Pathology, The First Affiliated Hospital of Guangxi Medical University, 6 Shuangyong Road, Nanning, Guangxi Zhuang Autonomous Region 530021, P.R. China E-mail: chengang@gxmu.edu.cn

${ }^{*}$ Contributed equally

Abbreviations: AUC, area under the curve; BP, biological process; CC, cellular component; CI, confidence interval; DEGs, differentially expression genes; DOR, diagnostic odds ratio; FISH, fluorescence in situ hybridization; GEO, Gene Expression Omnibus; GO, Gene Ontology; LUAD, lung adenocarcinoma; LA-MPE, LUAD-associated malignant pleural effusion; KEGG, Kyoto Encyclopedia of Genes and Genomes; MF, molecular function; miRNA/miR, microRNA; NAFLD, non-alcoholic fatty liver disease; NLR, negative likelihood ratio; NSCLC, non-small cell lung cancer; PLR, positive likelihood ratio; PPI, protein-protein interaction; ROC, receiver operating characteristic; RT-qPCR, reverse transcription-quantitative polymerase chain reaction; SMD, standard mean difference; TCGA, The Cancer Genome Atlas; THPA, The Human Protein Atlas

Key words: microRNA-198-5p, reverse transcription-quantitative PCR, expression, lung adenocarcinoma, target genes, bioinformatics, microarray
PCR (RT-qPCR) in 101 paired LUAD and adjacent normal lung tissues. Subsequently, the miR-198-5p expression level was determined from microarray data from the Gene Expression Omnibus, ArrayExpress and by meta-analyses. Furthermore, the target mRNAs of miR-198-5p from 12 miRNA-mRNA predictive tools were intersected with The Cancer Genome Atlas (TCGA)-based differentially expressed genes. In addition, Gene Ontology annotation and Kyoto Encyclopedia of Genes and Genomes (KEGG) pathway analysis were conducted to determine the possible mechanism of miR-198-5p in LUAD. The Search Tool for the Retrieval of Interacting Genes/Proteins database was employed to construct a protein-protein interaction network among the potential target genes of miR-198-5p. The results showed that miR-198-5p expression was lower in LUAD tissues than in adjacent non-cancerous lung tissues $(4.469 \pm 2.495$ vs. $5.301 \pm 2.502 ; \mathrm{P}=0.015)$. Meta-analyses, including the data from the present study and online microarray data, also verified the downregulation of miR-198-5p in 584 cases of LUAD. The expression of miR-198-5p was associated with the age, blood vessel invasion, Tumor-Node-Metastasis stage, and lymph node metastasis of patients with LUAD and served as an independent prognostic factor for survival. The hub genes of miR-198-5p were upregulated in LUAD, according to TCGA and The Human Protein Atlas. For the KEGG pathway analysis, the most enriched KEGG pathway was the p53 signaling pathway $\left(\mathrm{P}=1.42 \times 10^{-6}\right)$. These findings indicated that the downregulation of miR-198-5p may play a pivotal role in the development of LUAD by targeting various signaling pathways.

\section{Introduction}

Lung cancer, one of the most common malignancies and the leading cause of cancer-associated death in the USA and China over the past five years, mainly manifests in the form of non-small cell lung cancer (NSCLC) (1-4). A lack of specific early symptoms often leads to a delay in the diagnosis and treatment of patients with NSCLC. Thus, despite notable progress, including minimally invasive techniques, stereotactic radiotherapy, targeted therapy and immunotherapy, the five-year survival rate of lung cancer is estimated to be merely 
$16 \%$ (5). Lung adenocarcinoma (LUAD) is the major subtype of NSCLC and constituted $~ 50 \%$ of all lung cancer cases (43\% in men and 52\% in women) in the USA between 1992 and 2013 (6). Currently, the mechanism of LUAD initiation and progression is not entirely clear. Thus, there is a need to identify more effective biomarkers of this disease.

MicroRNAs (miRs/miRNAs) are a group of single-stranded, non-protein-coding RNAs that are 19-25 nucleotides in length, and regulate genes by base-pairing to the 3 '-untranslated regions of their target genes (7). Some miRNAs were demonstrated to be expressed in LUAD tissues and to be associated with survival in patients $(8,9)$. miR-198-5p was reported to be involved in various human malignancies (10-12), such as NSCLC (13). Yang et al (13) reported that miR-198-5p inhibits the development of LUAD in vitro and in vivo by regulating fibroblast growth factor receptor 1; however, the exact role of miR-198-5p in LUAD and its underlying mechanism remain poorly understood.

Aiming to discover the clinical value of miR-198-5p in LUAD, its expression level was detected using RT-qPCR. The associations between miR-198-5p expression and the clinicopathological characteristics of patients with LUAD, including overall survival (OS) time, were analyzed. Furthermore, meta-analyses based on microarray or miRNA-sequencing data from Gene Expression Omnibus (GEO), ArrayExpress and The Cancer Genome Atlas (TCGA) were performed. Finally, the target mRNAs of miR-198-5p were examined to explore the potential signaling pathways involved. A summary of the study design is illustrated in Fig. 1.

\section{Materials and methods}

Patients. A total of 101 patients with LUAD were enrolled in the study between January 2014 and December 2016 at The First Affiliated Hospital of Guangxi Medical University (Nanning, China), whose Medical Ethics Committee approved this study (approval no. 2015 KY-E-041). Each participant signed an informed consent form. The inclusion criteria were as follows: i) Diagnosed with LUAD with complete pathological reports; ii) both tumor and adjacent non-tumor tissues were available; iii) staging information was complete; iv) age $\leq 80$ years; and v) home address and telephone number were available. The exclusion criteria were as follows: i) Any history of malignancies other than LUAD; and ii) antitumor therapy before pathological tissues were obtained. Paraffin-embedded samples of tumor tissues and matched adjacent normal tissues were collected from each patient. All samples were fixed with $10 \%$ neutral formalin for $16-24 \mathrm{~h}$ at room temperature prior to paraffin embedding. The staging was classified according to the 7th edition of the UICC-AJCC Tumor-Node-Metastasis staging system (14). The median patient follow-up time was 28.4 months. OS time was defined as the period between pathological diagnosis and death.

$R T-q P C R$. The miRNeasy FFPE kit (Qiagen $\mathrm{GmbH}$ ) was used to extract total RNA from the aforementioned samples, and NanoDrop 2000 (Thermo Fisher Scientific, Inc.) was used to measure the concentration of RNA. Mir- $\mathrm{X}^{\mathrm{TM}}$ miRNA qRT-PCR TB Green ${ }^{\circledR}$ Kit (Takara Bio, Inc.) was used to synthesize cDNA from total RNA. miR-191-5p was considered as an internal reference due to its stable expression in lung

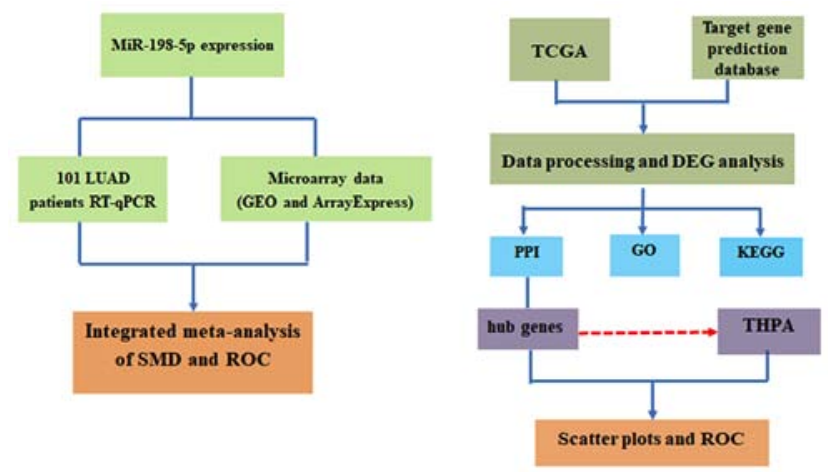

Figure 1. Flow diagram of the study design. miR, microRNA; LUAD, lung adenocarcinoma; RT-qPCR, reverse transcription-quantitative PCR; GEO, Gene Expression Omnibus; SMD, standard mean difference; ROC, receiver operating characteristic; TCGA, The Cancer Genome Atlas; DEG, differentially expression gene; PPI, protein-protein interaction; GO, Gene Ontology; KEGG, Kyoto Encyclopedia of Genes and Genomes; THPA, The Human Protein Atlas.

cancer and normal lung tissues (15). The primer sequence for miR-198-5p was 5'-CAACGGAAUCCCAAAAGCAGCU-3' and the sequence for miR-191-5p was 5'-GGUCCAGAGGGG AGAUAGGUUC-3'. PCR was performed with an Applied Biosystems 7900 PCR system (Thermo Fisher Scientific, Inc.) using SYBR Premix Ex Taq (Takara Bio, Inc.). The reaction conditions of the PCR were as follows: $98^{\circ} \mathrm{C}$ for $10 \mathrm{~min}$, followed by 42 cycles of $3 \mathrm{sec}$ at $98^{\circ} \mathrm{C}$ and $30 \mathrm{sec}$ at $55^{\circ} \mathrm{C}$. The $2^{-\Delta \Delta \mathrm{Cq}}$ method was utilized to calculate the relative expression of miR-198-5p (16).

Database searches. A LUAD-associated microarray search was performed in the GEO (www.ncbi.nlm.nih.gov/geo) (17) and ArrayExpress (www.ebi.ac.uk/arrayexpress) (18) databases on December 1, 2017. The following terms were used: ['lung' OR 'pulmonary' OR 'respiratory' OR 'bronchioles' OR 'bronchi' OR 'alveoli' OR 'pneumocytes' OR 'air way' $(\mathrm{MeSH})$ ] and ['cancer' OR 'carcinoma' OR 'tumor' OR 'neoplas* OR malignan* squamous cell carcinoma' OR 'adenocarcinoma' (MeSH)] or/and ['MicroRNA' OR 'miRNA' OR 'MicroRNA' OR 'Small Temporal RNA' OR 'noncoding RNA' OR 'ncRNA' OR 'small RNA' (MeSH)].

Studies that met the following criteria were included: First, LUAD tissues were available in the LUAD group, and normal lung tissues were available in the control group. Secondly, $>5$ samples were available in each group. Thirdly, the miR-198-5p level was available for the LUAD and control groups. Fourthly, only human samples were included. Because the microarray data obtained from the two databases were identical, GEO was chosen for the subsequent analyses. The expression data of miR-198-5p were $\log _{2}$-tranformed for further analysis.

The PubMed (www.ncbi.nlm.nih.gov/pubmed), Web of Science (clarivate.com/products/web-of-science), Science Direct (www.sciencedirect.com), Ovid (http://www.ovid. com/), LILACS (lilacs.bvsalud.org/en), Wiley Online Library (www.onlinelibrary.wiley.com/), EMBASE (ttuhsc.libguides. com/embase), and CNKI (cnki.net) databases were also searched for publications that discussed miR-198-5p expression in LUAD, and only one relevant article was retrieved (19). In addition, TCGA miRNA data associated with LUAD and 
Table I. Association between microRNA-198-5p expression and the clinicopathological features in LUAD.

\begin{tabular}{|c|c|c|c|c|}
\hline Clinicopathological feature & $\mathrm{n}$ & Mean \pm SD & Statistical value ${ }^{a}$ & P-value \\
\hline Tissue & & & -2.477 & 0.015 \\
\hline LUAD & 101 & $4.469 \pm 2.495$ & & \\
\hline Non-tumor & 101 & $5.301 \pm 2.502$ & & \\
\hline Sex & & & -0.091 & 0.928 \\
\hline Male & 56 & $4.448 \pm 2.103$ & & \\
\hline Female & 45 & $4.494 \pm 2.937$ & & \\
\hline Age & & & -2.727 & 0.008 \\
\hline$<60$ & 41 & $3.732 \pm 1.781$ & & \\
\hline$\geq 60$ & 60 & $4.972 \pm 2.787$ & & \\
\hline Smoking state & & & -1.147 & 0.258 \\
\hline No & 26 & $3.769 \pm 1.754$ & & \\
\hline Yes & 18 & $4.439 \pm 2.106$ & & \\
\hline Size & & & 0.689 & 0.493 \\
\hline$\leq 3 \mathrm{~cm}$ & 53 & $4.632 \pm 2.619$ & & \\
\hline$>3 \mathrm{~cm}$ & 48 & $4.288 \pm 2.365$ & & \\
\hline EGFR mutation & & & -0.096 & 0.924 \\
\hline Wild-type & 20 & $3.835 \pm 1.968$ & & \\
\hline Mutation & 13 & $3.900 \pm 1.792$ & & \\
\hline EGFR amplification & & & 0.348 & 0.730 \\
\hline No & 21 & $3.948 \pm 2.009$ & & \\
\hline Yes & 12 & $3.708 \pm 1.679$ & & \\
\hline Vascular invasion & & & 2.159 & 0.033 \\
\hline No & 70 & $4.819 \pm 2.642$ & & \\
\hline Yes & 31 & $3.677 \pm 1.941$ & & \\
\hline TNM stage & & & 2.391 & 0.019 \\
\hline I-II & 44 & $5.154 \pm 2.838$ & & \\
\hline III-IV & 57 & $3.939 \pm 2.069$ & & \\
\hline LNM & & & 2.105 & 0.038 \\
\hline No & 45 & $5.042 \pm 2.781$ & & \\
\hline Yes & 56 & $4.008 \pm 2.156$ & & \\
\hline EGFR protein expression & & & 0.091 & 0.928 \\
\hline Low & 22 & $3.882 \pm 1.994$ & & \\
\hline High & 11 & $3.818 \pm 1.691$ & & \\
\hline MET expression & & & -0.510 & 0.614 \\
\hline Low & 20 & $3.725 \pm 1.808$ & & \\
\hline High & 13 & $4.069 \pm 2.023$ & & \\
\hline Grading $^{\mathrm{b}}$ & & & 0.448 & 0.640 \\
\hline I & 17 & $4.979 \pm 3.073$ & & \\
\hline II & 61 & $4.328 \pm 2.242$ & & \\
\hline III & 23 & $4.465 \pm 2.495$ & & \\
\hline
\end{tabular}

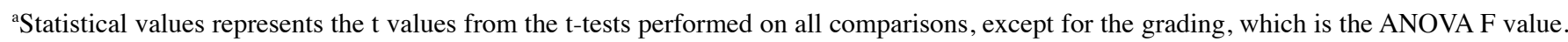
$\mathrm{F}$ value is defined as the ratio of between group variance to within group variance. ${ }^{\mathrm{b}} \mathrm{Grading}$ for lung adenocarcinoma included three categories (61): I represents well-differentiated, II represents moderately differentiated, and III represents poorly-differentiated adenocarcinoma. miRNA, microRNA; LUAD, lung adenocarcinoma; TNM, Tumor-Node-Metastasis; LNM, lymph node metastasis; EGFR, epidermal growth factor receptor; MET, hepatocyte growth factor receptor.

non-cancerous samples from The University of California Santa Cruz Xena (xena.ucsc.edu) was downloaded. Two cohorts were obtained, each with $<3$ samples available. Therefore, due to an insufficient number of patients with LUAD or control cases in the miR-198-5p expression data, TCGA was not included in the investigation. 
A

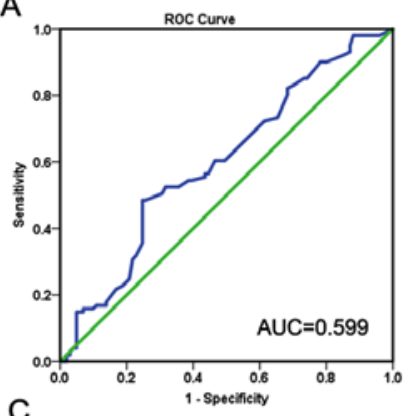

C

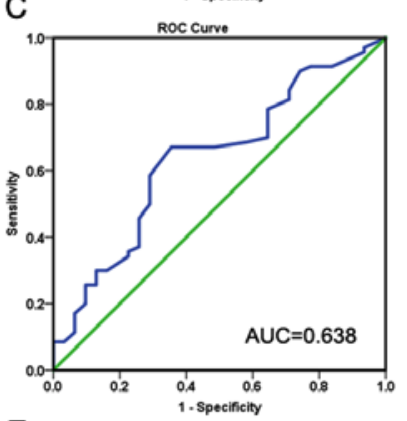

$\mathrm{E}$

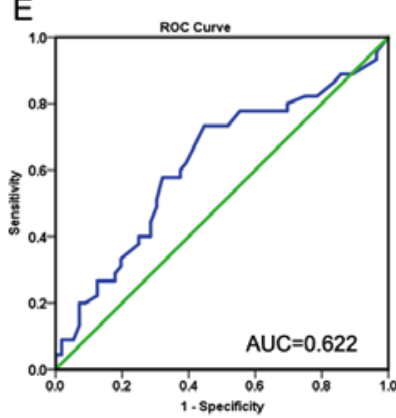

B
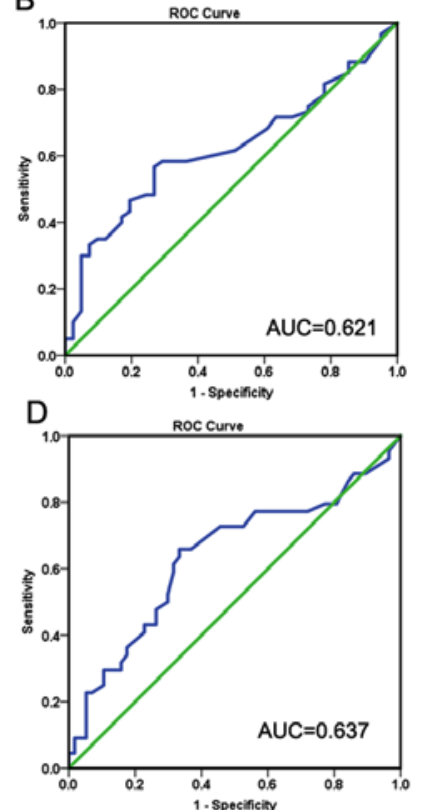

$F$

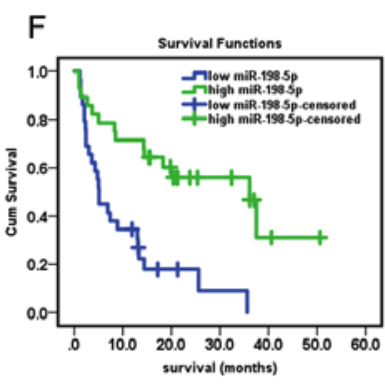

Figure 2. Association between the miR-198-5p expression level and the different clinicopathological factors in LUAD, with corresponding ROC curves. Comparison of mir-198-5p expression was made according to (A) LUAD and control groups; (B) age; (C) vascular invasion; (D) TNM stage; and (E) LNM.(F) Survival analysis in patients in the low- and high-miR-198-5p expression groups. miR, microRNA; LUAD, lung adenocarcinoma; ROC, receiver operating characteristic; TNM, Tumor-Node-Metastasis; LNM, lymph node metastasis.

Data preprocessing and differentially expressed gene (DEG) analysis. The DESeq R package (version 1.18.1) (20) was employed to process the original gene expression data of mRNA in LUAD that was obtained from TCGA (ID: TCGA-LUAD). The DEGs between the LUAD and non-cancer groups were identified, and upregulated DEGs with a fold change (FC) $>2$ were subjected to further analysis. As miR-198-5p exhibited a lower expression level in LUAD tissues, downregulated DEGs were not selected for subsequent bioinformatics analyses.

Identification of potential target genes. The potential targets of miR-198-5p were predicted using miRWalk (version 2.0; zmf. umm.uni-heidelberg.de/mirwalk2), a comprehensive database that includes 12 online predictive tools: microT-CDS, microT4, miRanda, miRBridge, miRDB, miRMap, miRNAMap, PICTAR2, PITA, RNA22, RNAhybrid and TargetScan (21). Genes that appeared in more than two databases were cross-referenced with the upregulated DEGs identified in TCGA analysis. The overlapping genes were considered to be potential target genes of miR-198-5p in LUAD.

Table II. Univariate analysis based on the follow-up of patients with lung adenocarcinoma.

\begin{tabular}{|c|c|c|c|c|}
\hline \multirow[b]{2}{*}{ Variable } & \multicolumn{4}{|c|}{ Overall survival time (months) } \\
\hline & P-value & HR & $\begin{array}{l}\text { Lower } \\
\text { limit }\end{array}$ & $\begin{array}{l}\text { Upper } \\
\text { limit }\end{array}$ \\
\hline \multicolumn{5}{|l|}{ Sex } \\
\hline Male vs. female & 0.456 & 0.779 & 0.404 & 1.502 \\
\hline \multicolumn{5}{|l|}{ Age } \\
\hline$<60$ vs. $\geq 60$ & 0.901 & 0.956 & 0.470 & 1.945 \\
\hline $\begin{array}{l}\text { Size } \\
\leq 3 \mathrm{~cm} \text { vs. } 3 \mathrm{~cm}\end{array}$ & 0.318 & 1.396 & 0.725 & 2.691 \\
\hline $\begin{array}{l}\text { Vascular invasion } \\
\text { No vs. yes }\end{array}$ & 0.860 & 1.059 & 0.562 & 1.995 \\
\hline \multicolumn{5}{|l|}{ TNM stage } \\
\hline \multicolumn{5}{|l|}{ LNM } \\
\hline \multicolumn{5}{|l|}{ Grade } \\
\hline II vs. I & 0.699 & 1.213 & 0.456 & 3.233 \\
\hline III vs. I & 0.698 & 1.108 & 0.660 & 1.860 \\
\hline II vs. III & 0.754 & 0.892 & 0.438 & 1.817 \\
\hline \multicolumn{5}{|l|}{$\begin{array}{l}\text { miR-198-5p } \\
\text { expression }\end{array}$} \\
\hline High vs. low & $<0.001$ & 0.272 & 0.133 & 0.555 \\
\hline
\end{tabular}

HR, hazard ratio; TNM, Tumor-Node-Metastasis; LNM, lymph node metastasis; miR, microRNA.

Gene Ontology (GO), Kyoto Encyclopedia of Genes and Genomes (KEGG) and protein-protein interaction (PPI) maps. To discern the biological attributes of the putative target genes, GO (22) and KEGG enrichment analyses (23) were conducted using online functional annotation tools from the Database for Annotation, Visualization and Integrated Discovery (version 6.7; david.ncifcrf.gov) (24) and were completed using the ClusterProfiler $\mathrm{R}$ package with default threshold $(\mathrm{P}<0.05)$ (version 3.4.1; bioconductor. org/packages/release/bioc/html/clusterProfiler.html) (25). The functional network graph of the selected genes was further visualized. The PPI maps of the putative mRNAs were constructed using the Search Tool for the Retrieval of Interacting Genes/Proteins database (version 10.0; version10. string-db.org) with default settings.

Validation of the hub genes of miR-198-5p in LUAD based on TCGA and The Human Protein Atlas (THPA). In the PPI network, genes were selected whose edges (the number of the protein-protein interactions) ranked among the top 10, and were considered the hub genes of miR-198-5p in LUAD. If several genes had the same edges, the genes with higher $\log _{\mathrm{FC}}$ values were selected. Scatter plots and receiver operating characteristic (ROC) curves were generated to show the expression of each hub gene, based on TCGA. The insufficient 

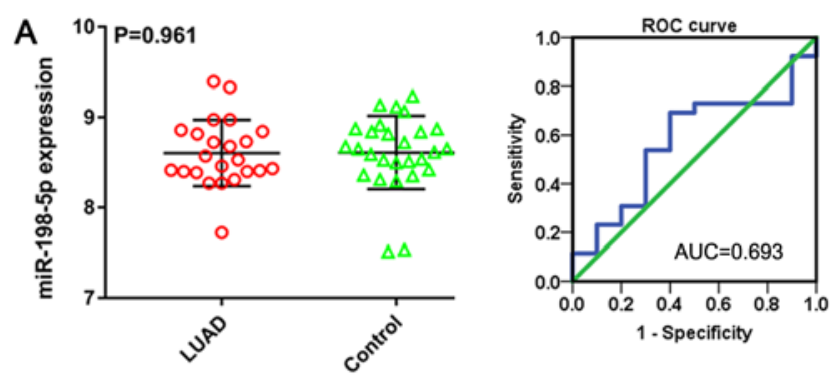

GSE14936

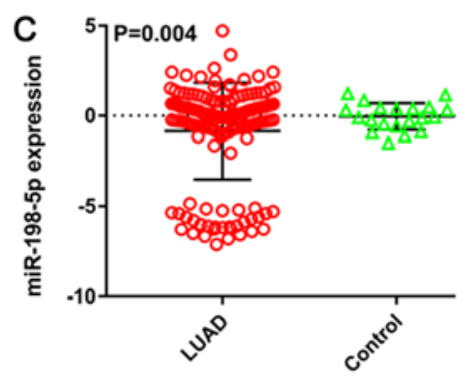

GSE48414
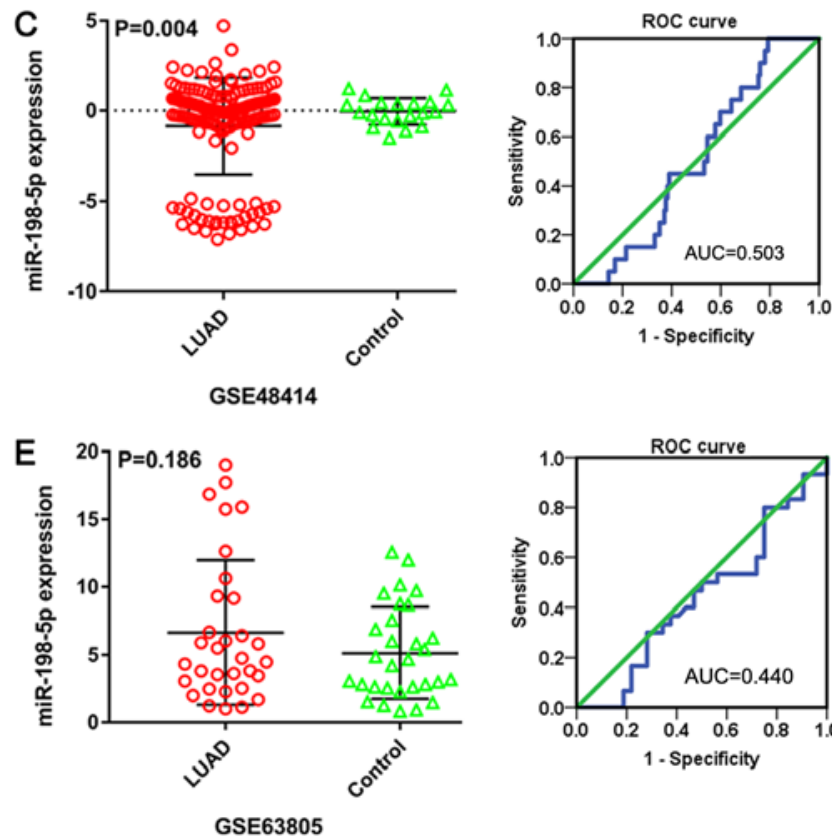

B

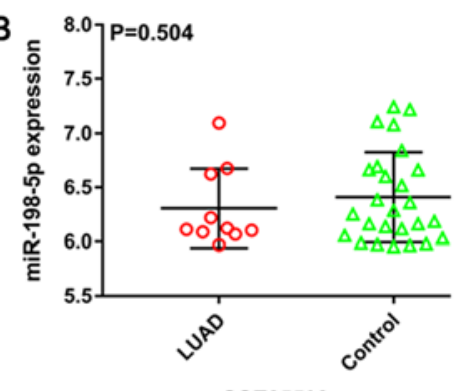

D
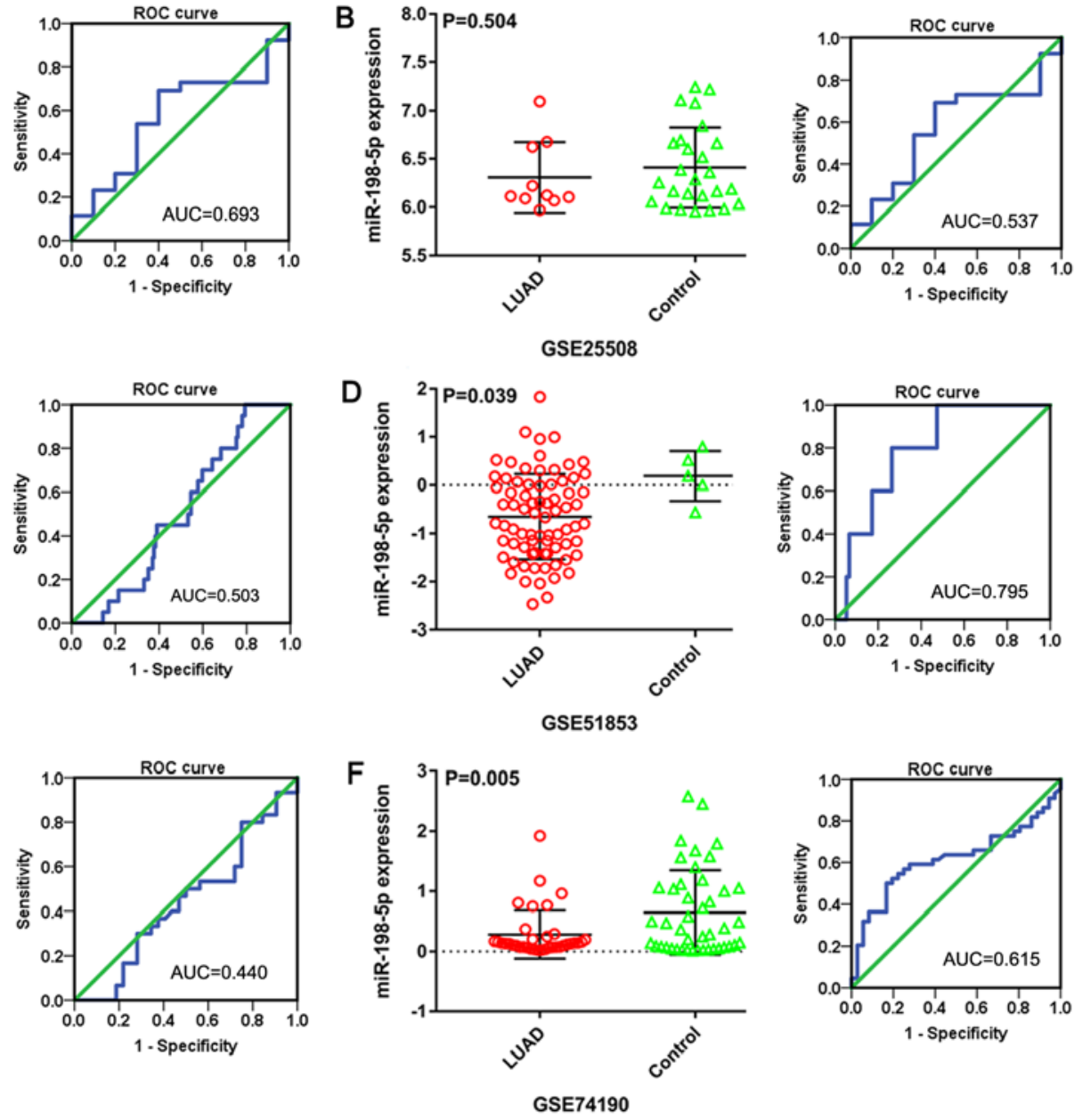

Figure 3. Scatter plots and ROC curves based on each Gene Expression Omnibus microarray dataset. (A) GSE14936; (B) GSE25508; (C) GSE48414; (D) GSE51853; (E) GSE63805; (F) GSE74190. miR, microRNA; LUAD, lung adenocarcinoma; ROC, receiver operating characteristic.

data related to miR-198-5p expression in TCGA restricted the analysis. Subsequently, the expression data of the hub genes at the protein level were downloaded from THPA (http://www. proteinatlas.org/), an open source database that provides data on the expression of proteins in a variety of human tissues. The expression of the hub genes was analzyed according to the staining intensity in THPA.

Statistical analysis. The quantitative data of miR-198-5p expression are presented as the mean \pm standard deviation. Student's t-test for independent samples, paired sample t-test, and one-way analysis of variance with Fisher's least significance difference post hoc test were performed in SPSS (version 19.0; IBM Corp.) to analyze the association between miR-198-5p expression and various clinicopathological characteristics. $\mathrm{P}<0.05$ was considered to indicate a statistically significant difference.

The sensitivity and specificity of mir-198-5p, as a diagnostic marker, was assessed by generating ROC curves, and calculating the area under the curve (AUC) using SPSS version 19.0.

The Kaplan-Meier method was used to estimate the OS rate of patients with LUAD, and the log-rank test was used to calculate the differences between groups. To evaluate the survival variables, univariate and multivariate (if applicable) Cox regression analyses were performed.
To evaluate the differential expression of miR-198-5p between LUAD tissues and normal tissues, data from GEO and ArrayExpress were subjected to a continuous variable meta-analysis in Stata 12 (StataCorp LP) and the results are presented as the overall standardized mean difference (SMD) and a 95\% confidence interval (CI). The fixed effects model was used first. Heterogeneity across studies was assessed using the $\chi^{2}$ test of $\mathrm{Q}$ and the $\mathrm{I}^{2}$ statistic. The existence of heterogeneity was defined as $\mathrm{P}<0.05$ or $\mathrm{I}^{2}>50 \%$, indicating that the random effects model was more suitable. The source of heterogeneity was detected by a sensitivity analysis, as described previously (26). Begg's test was employed to assess publication bias with the criterion of $\mathrm{P}<0.05$. Based on the included microarray, the ROC curves were plotted. Subsequently, a diagnostic meta-analysis was conducted with MetaDiSc 1.4 (http://www.hrc.es/investigacion/metadisc_en.htm). Heterogeneity was detected in this model by a meta-regression and a threshold effect analysis. Finally, the continuous variable meta-analysis was repeated following the inclusion of the in-house RT-qPCR data for comparison. The data from both the in-house RT-qPCR analysis and a previously published study that investigated miR-198-5p expression in LUAD-associated pleural effusion (19) were included and the diagnostic meta-analysis was subsequently repeated. 
A

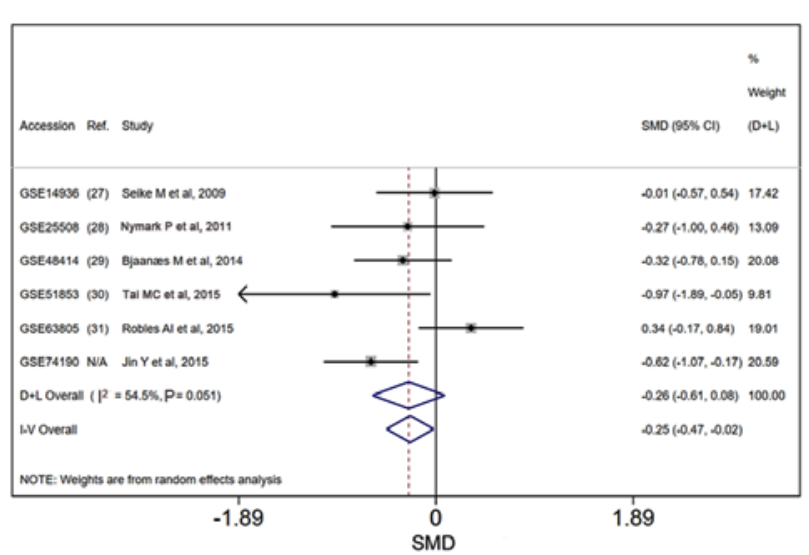

C

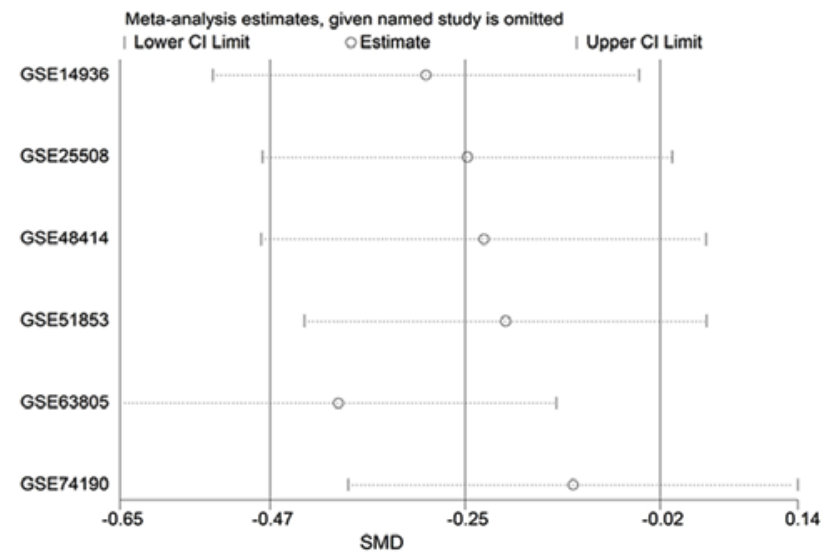

B

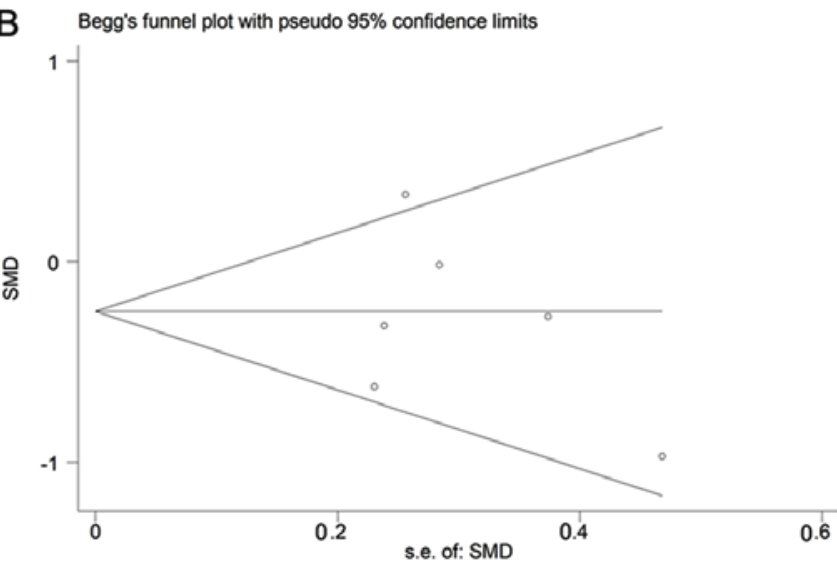

D

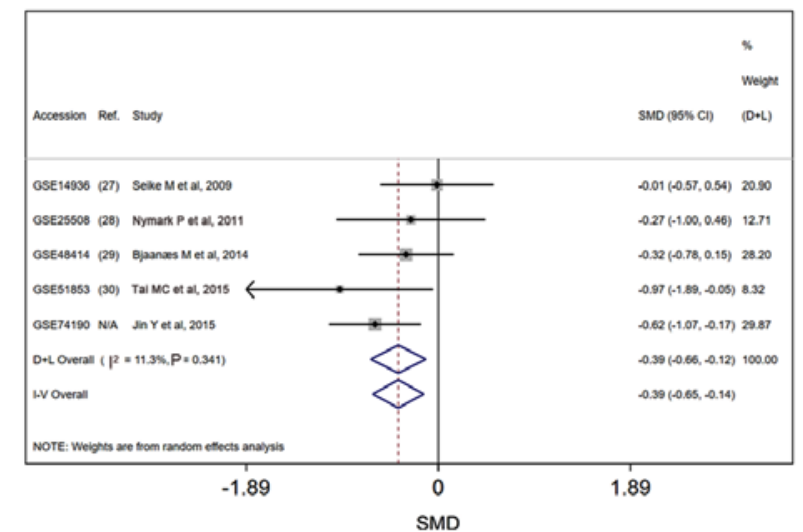

Figure 4. Continuous variable meta-analysis based on the included Gene Expression Omnibus microarray datasets. (A) Forest plot of the fixed effects model and the random effects model. (B) Begg's funnel plot. (C) Sensitivity analysis. (D) Forest plot based on the fixed effects model without GSE63805. CI, confidence interval; SMD, standard mean difference; N/A, not available; s.e., standard error; I-V, inverse variance method, D+L, Duckworth-Lewis method.

\section{Results}

Expression of miR-198-5p and diverse clinicopathological characteristics in LUAD. The clinicopathological characteristics of 101 patients with LUAD are listed in Table I. According to the results of the in-house RT-qPCR, the expression of miR-198-5p was lower in LUAD tissues than in corresponding adjacent non-tumor lung tissues $(4.469 \pm 2.495$ vs. $5.301 \pm 2.502$, respectively; $\mathrm{P}=0.015$; AUC, 0.599; Fig. 2A). However, an AUC value of 0.599 indicated that the performance of the ROC curve was not satisfactory. Subsequently, the expression of miR-198-5p in 101 LUAD tissues was compared for various clinicopathological factors. As shown in Table I, the samples from patients $<60$ years of age had lower expression of miR-198-5p compared with those from patients $\geq 60$ years of age $(3.732 \pm 1.781$ vs. $4.972 \pm 2.787$, respectively; $\mathrm{P}=0.008$; AUC, 0.621; Fig. 2B). The expression of miR-198-5p was also lower in tissues with vascular invasion compared with those without vascular invasion $(3.6774 \pm 1.9411$ vs. $4.819 \pm 2.642$, respectively; $\mathrm{P}=0.033 ; \mathrm{AUC}, 0.638$; Fig. $2 \mathrm{C}$ ), and samples from patients at Tumor-Node-Metastasis stages III-IV compared with those at stages I-II $(3.939 \pm 2.069$ vs. $5.154 \pm 2.838$, respectively; $\mathrm{P}=0.019$; AUC, 0.637; Fig. 2D). Moreover, a decrease in miR-198-5p expression was observed in samples with lymph node metastasis compared with those without lymph node metastasis $(4.008 \pm 2.156$ vs. $5.042 \pm 2.781$, respectively; $\mathrm{P}=0.038$; AUC, 0.622; Fig. 2E).
The entire cohort was divided into two groups (low and high mirR-198-5p expression) according to the median expression level of miR-198-5p in the LUAD tissues (median, 3.60). The survival rate of patients with high miR-198-5p expression was higher than those with low expression $(\mathrm{P}<0.001$; Fig. $2 \mathrm{~F})$. The univariate analysis revealed that the expression level of miR-198-5p served as an independent prognostic factor for OS time $(\mathrm{P}<0.001$; Table II). The multivariate analysis was not performed because other clinicopathological factors did not appear to be effective as prognostic predictors.

Meta-analysis based on LUAD microarray data. The expression pattern and diagnostic value of miR-198-5p was evaluated by a meta-analysis based on microarray data from the GEO datasets. A total of 483 samples from six GEO datasets (in which bodily fluid samples were not available) including GSE14936 (27), GSE25508 (28), GSE48414 (29), GSE51853 (30), GSE63805 (31) and GSE7419 were included to generate scatter plots and corresponding ROC curves (Fig. 3). No difference in miR-198-5p expression between LUAD and non-tumor lung tissues was observed in dataset GSE14936 ( $8.604 \pm 0.368$ vs. $8.609 \pm 0.406$, respectively; $\mathrm{P}=0.961$; AUC, 0.693 ; Fig. 3A) and dataset GSE25508 (6.308 \pm 0.366 vs. $6.409 \pm 0.416$, respectively; $\mathrm{P}=0.504$; AUC, 0.537; Fig. 3B). miR-198-5p showed a lower expression in LUAD than in non-tumor lung tissues in both the GSE48414 $(-0.844 \pm 2.673$ vs. $-0.040 \pm 0.731$, respectively; P=0.004; AUC, 0.503; Fig. 3C) 
A
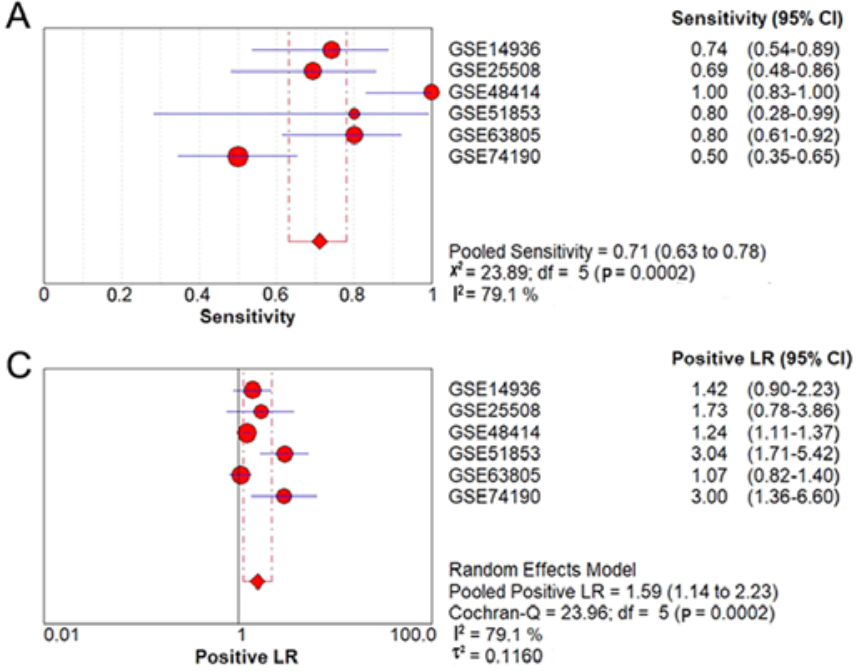

$\mathrm{E}$

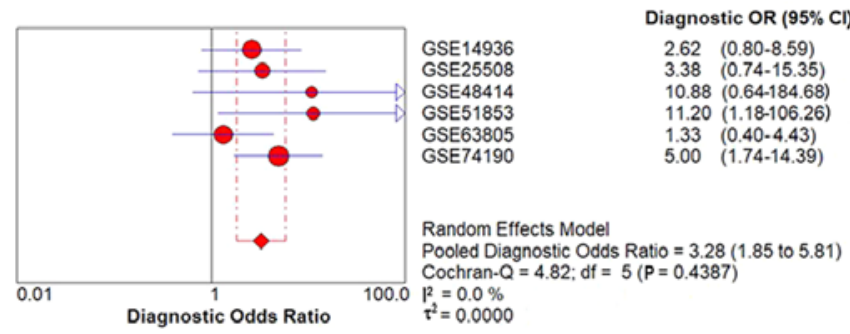

B
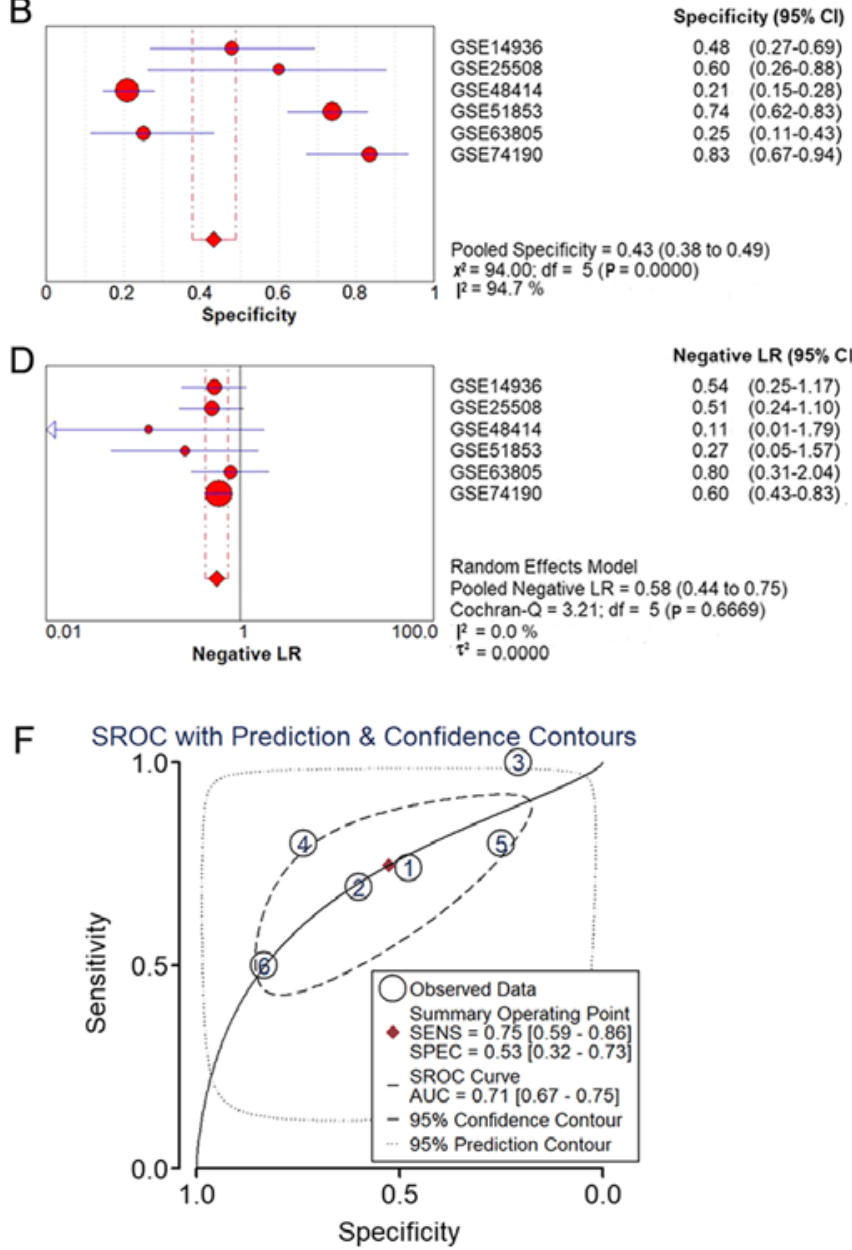

Figure 5. Diagnostic meta-analysis based on the included microarray datasets. (A) Sensitivity; (B) specificity; (C) positive LR; (D) negative LR; (E) diagnostic OR and (F) SROC curve of the included microarray datasets. LR, likelihood ratio; SROC, summarized receiver operating characteristic; OR, odds ratio; AUC, area under the curve; CI, confidence interval; SENS, sensitivity; SPEC, specificity.

and GSE51853 (-0.652 \pm 0.882 vs. $-0.189 \pm 0.516$, respectively; $\mathrm{P}=0.039$; AUC, 0.795; Fig. 3D) datasets. For the GSE63805 dataset, miR-198-5p expression in LUAD was similar to that in non-tumor lung tissues $(6.642 \pm 5.328$ vs. $5.132 \pm 3.397$, respectively; $\mathrm{P}=0.186$; AUC, 0.440; Fig. 3E). In contrast, the data from the GSE74190 dataset showed that miR-198-5p level was lower in LUAD than in non-tumor lung tissues $(0.281 \pm 0.400$ vs. 0.646 \pm 0.702 , respectively; $\mathrm{P}=0.005$; AUC, 0.615; Fig. 3F). miR-198-5p expression is illustrated in forest plots (Fig. 4A). An $\mathrm{I}^{2}$ value of $54.5 \%$ indicated the existence of heterogeneity across studies. The combined effect size was -0.25 (95\% CI, -0.47 to -0.02$)$ in the fixed effects models and $-0.26(95 \% \mathrm{CI},-0.61-0.08)$ in the random effects models. The funnel plots revealed no publication bias in this meta-analysis (P>0.05; Fig. 4B). Subsequently, a sensitivity analysis was performed to detect the source of heterogeneity. Each dataset was removed in turn and the meta-analysis was repeated to recalculate the heterogeneity. Upon removal of the GSE63805 dataset, the value of $\mathrm{I}^{2}$ sharply decreased to $11.3 \%$. No such decrease in $\mathrm{I}^{2}$ was observed when any other of the datasets was removed (Fig. 4C). GSE63805 was thus identified as the source of heterogeneity. The forest plot was then regenerated based on the fixed effects model, following the elimination of GSE63805 (Fig. 4D).
The diagnostic meta-analysis and the summarized ROC curve, based on the six included microarray datasets, are displayed in Fig. 5. The pooled sensitivity, specificity, positive likelihood ratio (PLR), negative likelihood ratio (NLR) and diagnostic odds ratio (DOR) were 0.71 (95\% CI, 0.63-0.78), 0.43 (95\% CI, 0.38-0.49), 1.59 (95\% CI, 1.14-2.23), 0.58 (95\% CI, 0.44-0.75) and 3.28 (95\% CI, 1.85-5.81), respectively, and the AUC was 0.71 .

Meta-analysis of miR-198-5p expression based on patients recruited for the present study and GEO LUAD data. The miR-198-5p expression data from the patients recruited for the present study were added to the data from the GEO datasets and the meta-analysis was repeated to determine the expression pattern in LUAD. Based on the fixed effects model, the combined effect size was -0.28 (95\% CI, -0.46-0.11) with $\mathrm{I}^{2}=46.5 \%$ (Fig. 6A). No publication bias was identified ( $\mathrm{P}>0.05$; Fig. 6B). Upon adding of the data from the present study and data that from a published article (PMID:23354517) (19), the pooled sensitivity, specificity, PLR, NLR and DOR were 0.62 (95\% CI, 0.56-0.58), 0.51 (95\% CI, 0.46-0.56), 1.67 (95\% CI, 1.18-2.36), 0.64 (95\% CI, 0.54-0.76) and 3.07 (95\% CI, 2.03-4.64), respectively (Fig. 7A-E), and the AUC was 0.74 (Fig. 7F). 

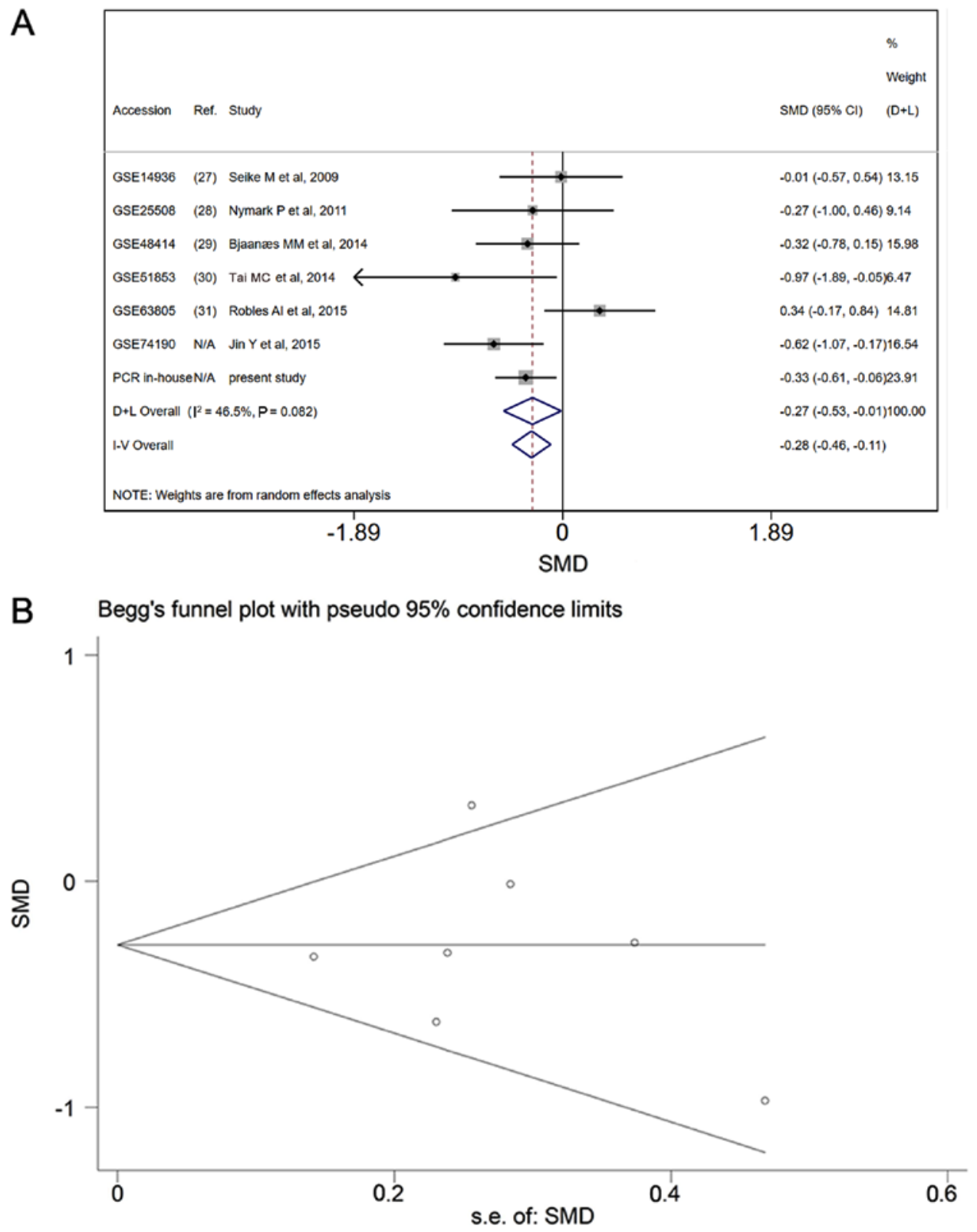

Figure 6. Continuous variable meta-analysis based on all included studies. (A) Forest plot based on the fixed effects model. (B) Begg's funnel plot. SMD, standard mean difference; CI, confidence interval; N/A, not available; s.e., standard error; I-V, inverse variance method, D+L, Duckworth-Lewis method.

GO and KEGG pathway analysis and construction of a PPI network. Overall, 197 overlapping genes were identified as potential targets of miR-198-5p in LUAD (Fig. 8A). The GO enrichment analysis included three categories: Biological process (BP; Fig. 8B), cellular component (CC; Fig. 8C) and molecular function (MF; Fig. 8D). The top five terms of each category are listed in Table III. The bubble plot in Fig. 9 displays the enriched GO terms. In the KEGG pathway analyses, the potential targets of miR-198-5p were most associated with the p53 signaling pathway $\left(\mathrm{P}=1.42 \times 10^{-6}\right.$; Table IV and Fig. 10). Other enriched terms included 'cell cycle', 'Parkinson's disease', 'Alzheimer's disease', 'oxidative phosphorylation', 'progesterone-mediated oocyte maturation', 'platinum drug resistance', and 'non-alcoholic fatty liver disease (NAFLD)'. The PPI network is shown in Fig. 11A with 66 representative nodes (ones with the largest number of edges) from 197 hub genes and the corresponding 123 edges and a PPI enrichment
$\mathrm{P}=1.55 \times 10^{-14}$. The selected hub genes were $\mathrm{G} 2 /$ mitotic-specific cyclin-B2 (CCNB2), Aurora kinase B (AURKB), cyclin-A2 (CCNA2), DNA topoisomerase 2- $\alpha$ (TOP2A), Aurora kinase A (AURKA), baculoviral IAP repeat-containing protein 5 (BIRC5), centromere protein N (CENPN), Rac GTPase-activating protein 1 (RACGAP1), protein regulator of cytokinesis 1 (PRC1) and cyclin-dependent kinase inhibitor 3 (CDKN3) (Fig. 11B).

Validation of the hub genes using TCGA and THPA. According to data from TCGA, the expression of all the selected hub genes was higher in the LUAD group than in the control group (all P<0.001), and the AUCs for CCNB2, AURKB, CCNA2, TOP2A, AURKA, BIRC5, CENPN, RACGAP1, PRC1 and CDKN3 were 0.968, 0.962, 0.965, 0.985, 0.947, 0.959, 0.830, $0.871,0.971$ and 0.936 , respectively (Fig. 12). The expression of the hub genes at the protein level, obtained from THPA, is 

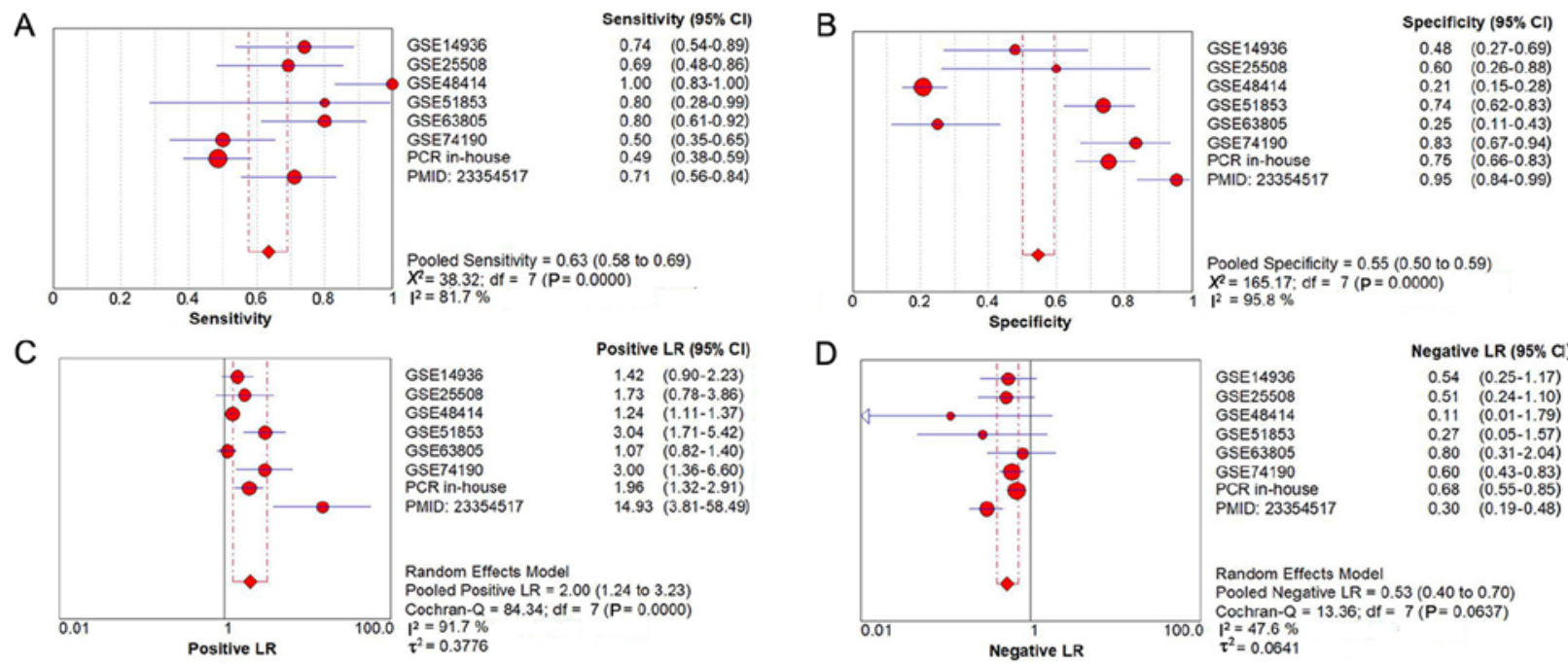

E
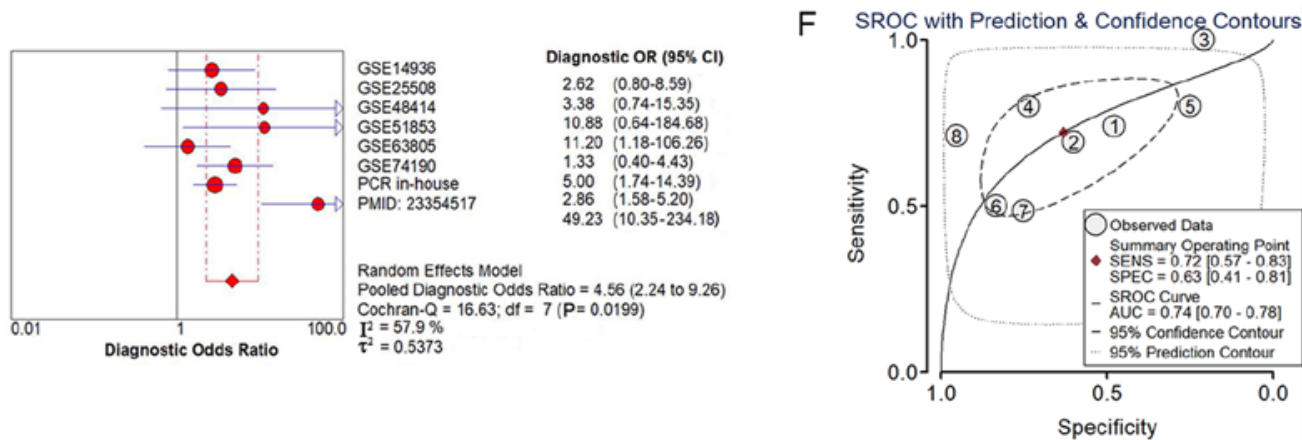

Figure 7. Diagnostic meta-analysis based on all included studies. (A) Sensitivity; (B) specificity; (C) positive LR; (D) negative LR; (E) diagnostic OR; (F) SROC curve of all included studies. LR, likelihood ratio; CI, confidence interval; SROC, summarized receiver operating characteristic; OR, odds ratio; SENS, sensitivity; SPEC, specificity.

A

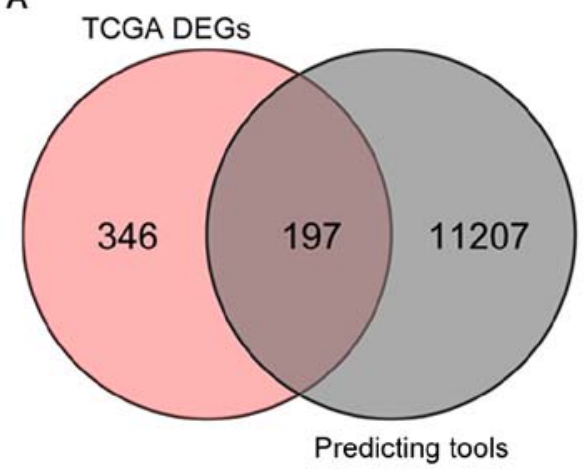

C

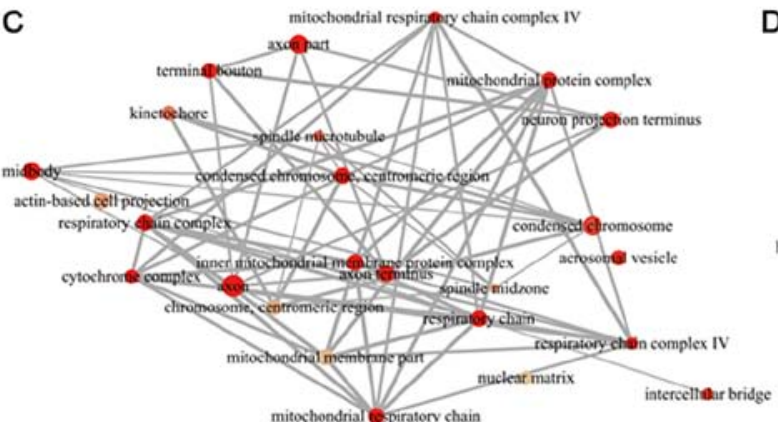

D
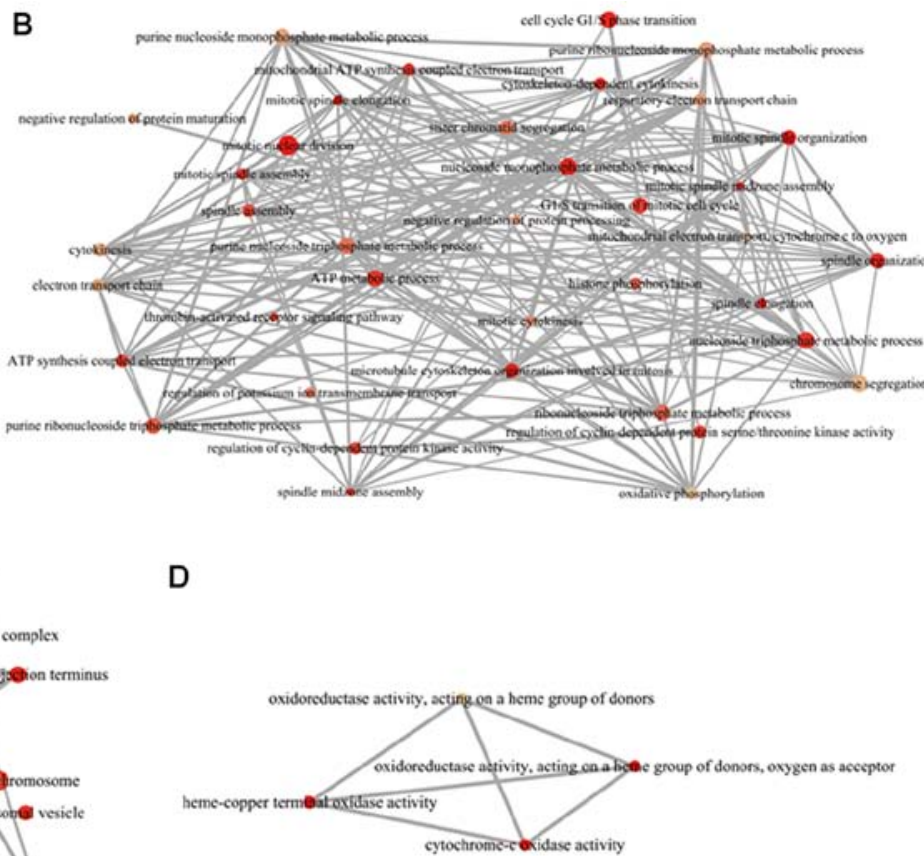

Figure 8. Venn diagram of overlapping genes and GO enrichment networks. (A) Venn diagram of the DEGs based on TCGA and predicting tools; GO enrichment networks of (B) biological processes; (C) cellular components; and (D) of molecule functions. GO, Gene Ontology; TCGA, The Cancer Genome Atlas; DEGs, differentially expressed genes. 


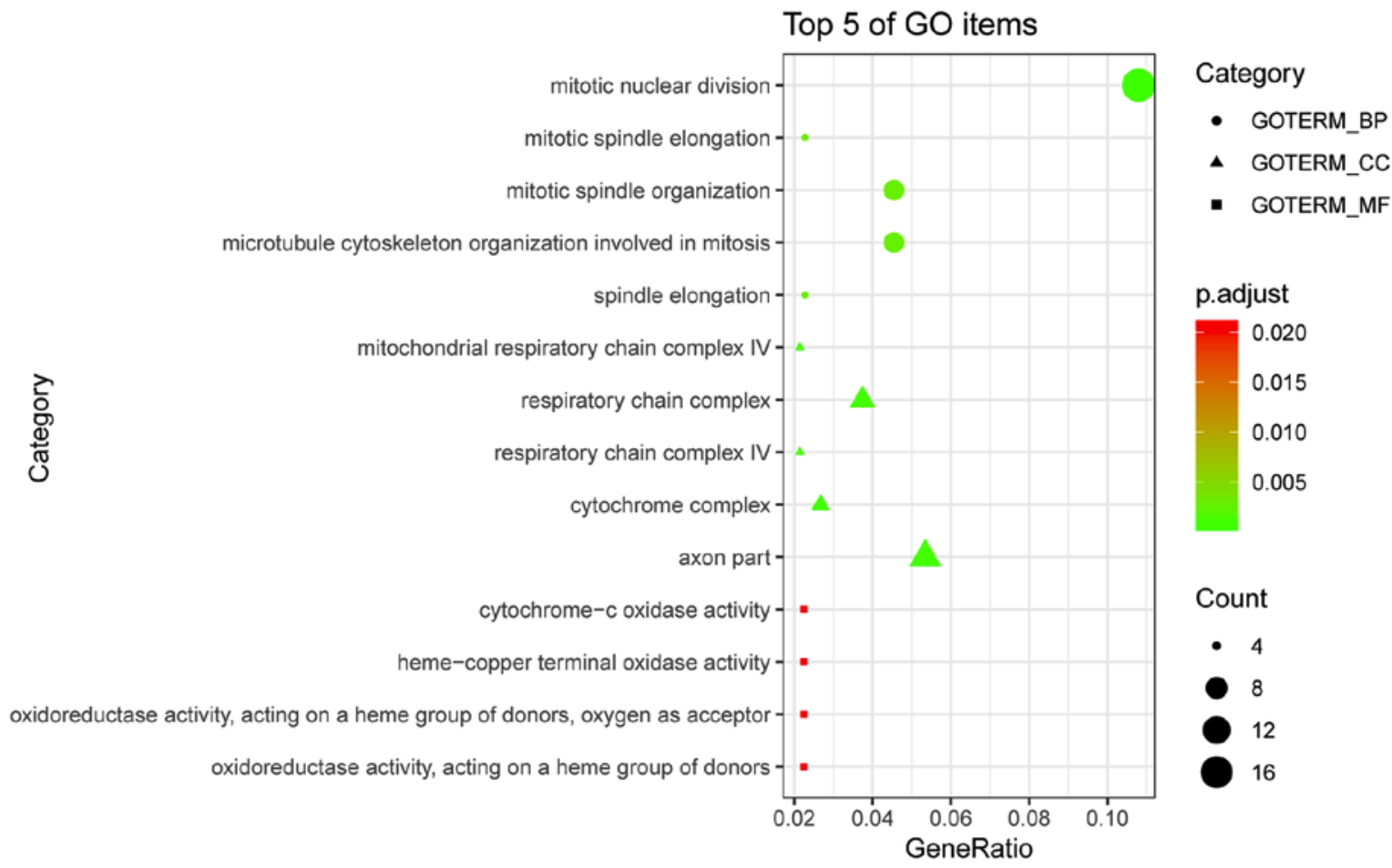

Figure 9. Bubble plot displaying the five most enriched GO, BP, CC and MF terms. GO, Gene Ontology; BP, biological process; CC, cellular component; MF, molecule function.

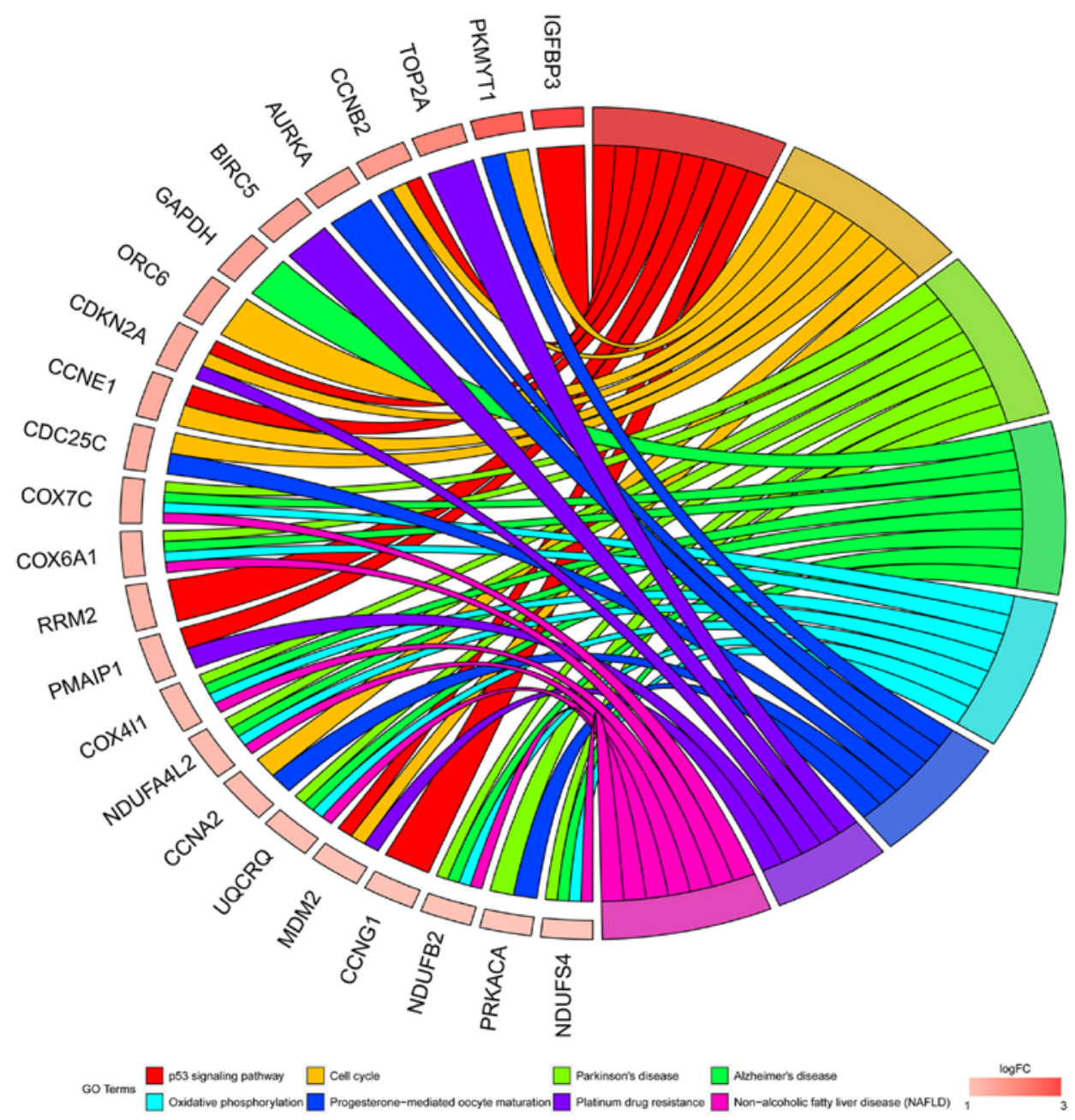

Figure 10. Chord plot displaying the result of the Kyoto Encyclopedia of Genes and Genomes pathway analysis. FC, fold change. 
A

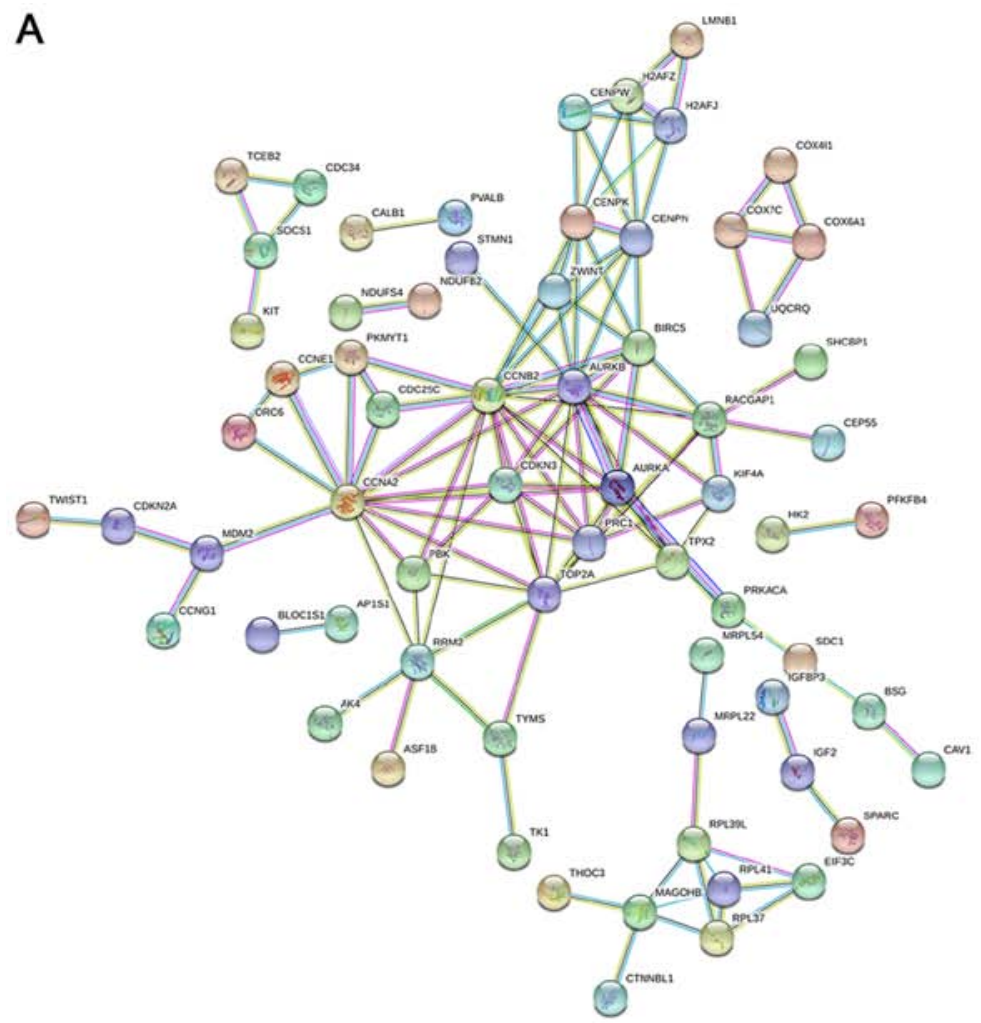

B

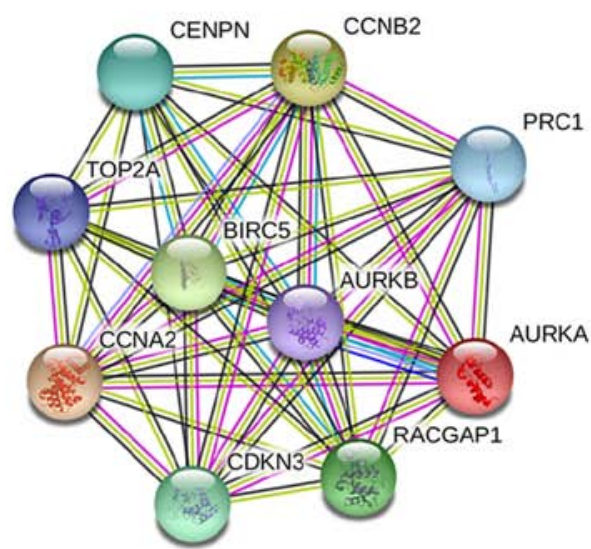

Figure 11. PPI maps based on the putative target genes and the representative hub genes of microRNA-198-5p in lung adenocarcinoma. PPI networks based on (A) the putative target genes; (B) 10 selected hub genes. PPI, protein-protein interaction.

displayed in Fig. 13. Eight of the ten hub genes were upregulated in LUAD tissues, whereas the expression data of CENPN and CDKN3 were not available in the THPA database.

\section{Discussion}

In the present study, the expression of miR-198-5p in LUAD was determined by in-house RT-qPCR on samples from 101 patients. Subsequently, a continuous variable meta-analysis based on microarray data was performed to validate this expression pattern. The results of the RT-qPCR and the microarray data were then integrated into a cohort of 584 cases, which enabled direct comparison. Comprehensive bioinformatics analyses were performed to explore the underlying pathways of miR-198-5p in LUAD. In addition, the expression data of the potential targets of miR-198-5p were acquired from TCGA and THPA. By comprehensively analyzing the results of the in-house RT-qPCR, microarray data mining and meta-analysis, it was found that miR-198-5p was differentially expressed in LUAD tissues and non-tumor lung tissues. Moreover, the downregulation of miR-198-5p in LUAD tissues was associated with multiple clinicopathological characteristics and served as an independent prognostic factor. In contrast, the potential target genes of miR-198-5p that were predicted by online tools were overexpressed in LUAD, according to TCGA and THPA data.

It has been previously observed that miR-198-5p is involved in multiple physiological processes, and its dysfunction can lead to various diseases. This miR was upregulated in both glomerular and tubulointerstitial regions of patients with lupus nephritis, indicating its participation in autoimmunity (32). In the tissues of fetuses with anencephaly, miR-198-5p was highly expressed and participated in the pathogenesis of anencephaly by regulating genes in a protein interaction network (33); however, downregulation of miR-198-5p was observed in respiratory syncytial virus infection and preeclampsia $(34,35)$. In Parkinson's disease, the expression level of miR-198-5p has been reported to be low and this mRNA is involved in several neurodegenerative pathways (36). Its upregulation in several malignant diseases, including esophageal cancer (37), multiple myeloma (38), pancreatic ductal adenocarcinoma (39), retinoblastoma (40) and tongue squamous cell carcinoma (41) was also reported. Moreover, the expression of miR-198-5p was negatively correlated with patient survival (37-39); however, miR-198-5p inhibits the development of certain types of cancer (42). In certain studies, downregulation of miR-198-5p was observed in prostate cancer (11), osteosarcoma (42), glioblastoma (43), as well as in certain malignancies of the digestive system, such as colorectal cancer (44), gastric cancer (45), pancreatic cancer (46) and hepatocellular carcinoma (47).

miR-198-5p was reported to enhance chemotherapy sensitivity in glioblastoma by targeting $\mathrm{O}^{6}$-methylguanine-DNA methyltransferase (43) or by directly inhibiting cell proliferation and migration (12), vital processes for tumorigenesis. In addition, there is evidence indicating that miR-198-5p participates in anti-tumor immunity, since its overexpression in the $\mathrm{CD}^{+} \mathrm{T}$ cells of patients with renal cell carcinoma leads to immune dysfunction via the targeting of Janus kinase 3 and myeloid cell leukemia 1 (48).

In a previous study, decreased expression of miR-198-5p was observed in lung squamous cell carcinoma tissues (SMD, 
Table III. Five most enriched terms in BP, CC and MF based on the GO analysis.

\begin{tabular}{lllrr}
\hline Category & \multicolumn{1}{c}{ ID } & \multicolumn{1}{c}{ Description } & Count, n & P-value \\
\hline GOTERM_BP & GO:0007067 & Mitotic nuclear division & 19 & $2.49 \times 10^{-7}$ \\
GOTERM_BP & GO:0000022 & Mitotic spindle elongation & 4 & $3.47 \times 10^{-6}$ \\
GOTERM_BP & GO:0007052 & Mitotic spindle organization & 8 & $4.62 \times 10^{-6}$ \\
GOTERM_BP & GO:1902850 & $\begin{array}{l}\text { Microtubule cytoskeleton organization } \\
\text { involved in mitosis }\end{array}$ & 8 & $4.62 \times 10^{-6}$ \\
& & Spindle elongation & 4 & $5.16 \times 10^{-6}$ \\
GOTERM_BP & GO:0051231 & Mitochondrial respiratory chain complex IV & 4 & $5.09 \times 10^{-6}$ \\
GOTERM_CC & GO:0005751 & Respiratory chain complex & 4 & $8.48 \times 10^{-6}$ \\
GOTERM_CC & GO:0098803 & Respiratory chain complex IV & 5 & $1.01 \times 10^{-5}$ \\
GOTERM_CC & GO:0045277 & Cytochrome complex & 10 & $1.08 \times 10^{-5}$ \\
GOTERM_CC & GO:0070069 & Axon part & 4 & $1.41 \times 10^{-5}$ \\
GOTERM_CC & GO:0033267 & Cytochrome-c oxidase activity & $4.70 \times 10^{-4}$ \\
GOTERM_MF & GO:0004129 & Heme-copper terminal oxidase activity & 4 & $1.70 \times 10^{-4}$ \\
GOTERM_MF & GO:0015002 & oxidoreductase activity, acting on a Heme & 4 & $1.96 \times 10^{-4}$ \\
GOTERM_MF & GO:0016676 & group of donors, oxygen as acceptor & & \\
GOTERM_MF & GO:0016675 & Oxidoreductase activity, acting on a heme & group of donors & \\
& & & & \\
\hline
\end{tabular}

BP, biological process; CC, cellular component; MF, molecular function; GO, Gene Ontology.

-0.34; 95\% CI, -0.71-0.04) (49). For LUAD, miR-198-5p initially received attention due to its differential expression between benign pleural effusion and LUAD-associated malignant pleural effusion (LA-MPE). Han et al $(19,50)$ reported decreased expression of miR-198-5p in LA-MPE, indicating its diagnostic potential for this condition. It was observed that miR-198-5p inhibits lung cancer cellular proliferation and induced apoptosis by regulating the expression of fibroblast growth factor receptor 1 (13).

The chemotherapeutic sensitivity of human LUAD cell line A549 was demonstrated to be positively regulated by miR-198-5p (13); however, miR-198-5p expression and the prognosis of patients with LUAD, in terms of OS, were not found to be associated. The in-house RT-qPCR performed in the present study revealed that miR-198-5p was downregulated in LUAD tissues. The continuous variable meta-analysis, which was based on microarray data, showed the same trend. Following the inclusion of the RT-qPCR data to the microarray data, no heterogeneity or publication bias was observed. Considering the unsatisfactory AUCs in the diagnostic meta-analysis, the diagnostic value of miR-198-5p in LUAD was not well-established. In addition, all the samples used for continuous variable meta-analysis were obtained from lung tissues rather than bodily fluids such as blood and urine, limiting the use of miR-198-5p as a diagnostic marker for LUAD. Surgery is the major mode of therapy for patients with early-stage (TNM stages I-II) LUAD, whereas patients with advanced-stage (TNM stages III-IV) LUAD rely mainly on other treatments, such as radiotherapy, chemotherapy, molecular targeted therapy and immunotherapy (51). Thus, the patients were divided into two groups (those at early stage and those at advanced stage) and lower expression of miR-198-5p was observed in samples from patients at stages III-IV compared with those at stages I-II.
Lung cancer is considered to be an age-associated disease, and multiple age-associated miRNAs have been reported to be expressed aberrantly in lung cancer (52). In the present study, the level of miR-198-5p was associated with age, whereas age and the prognosis of patients were not associated. Whether the prognosis of patients with LUAD was affected by other clinicopathological factors is worthy of further investigation. miR-198-5p expression was also associated with blood vessel invasion and lymph node metastasis in patients with LUAD. Moreover, the downregulation of miR-198-5p in LUAD tissues was associated with shorter survival time and served as an independent prognostic factor. Unfortunately, the primary focus was the influence of miR-198-5p on OS, and not all patients diagnosed with LUAD received further anticancer treatment at the hospital. Other characteristics, such as weight, height, underlying diseases and treatment modality, were not fully available, which further limited the study.

In the present study, 12 online miRNA-mRNA target prediction tools were applied to predict the target mRNAs of miR-198-5p, which had not yet been performed for LUAD, to the best of our knowledge. To further enhance the reliability of the study, the predicted targets of miR-198-5p in LUAD were cross-referenced with the DEGs in TCGA. As miR-198-5p was downregulated in LUAD, the upregulated DEGs were selected from TCGA for further analysis.

Regarding the potential underlying mechanism of miR-198-5p in LUAD, the bioinformatics analysis showed the p53 signaling pathway as the most enriched pathway, followed by cell cycle, Parkinson's disease, Alzheimer's disease, oxidative phosphorylation, progesterone-mediated oocyte maturation, platinum drug resistance and NAFLD. The p53 signaling pathway, Alzheimer's disease, oxidative phosphorylation, progesterone-mediated oocyte maturation, 

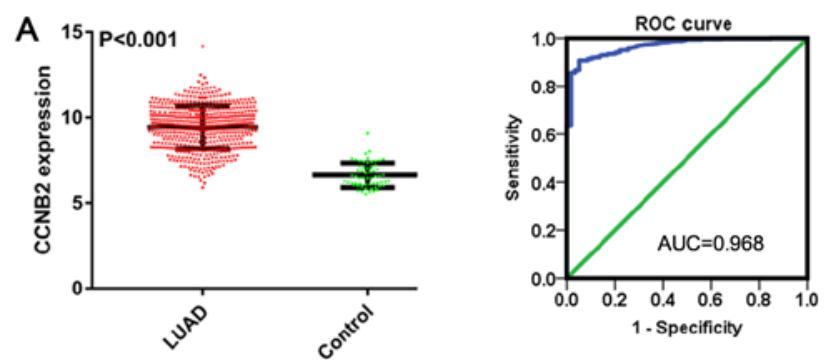

C

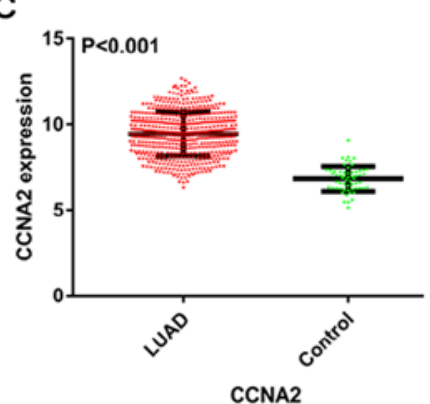

E

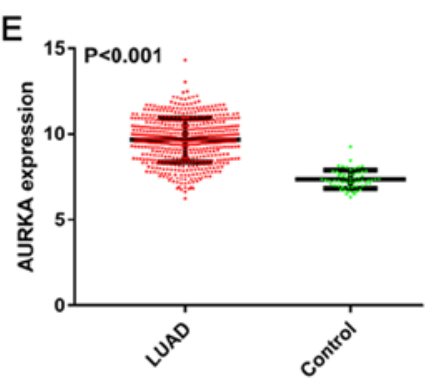

AURKA

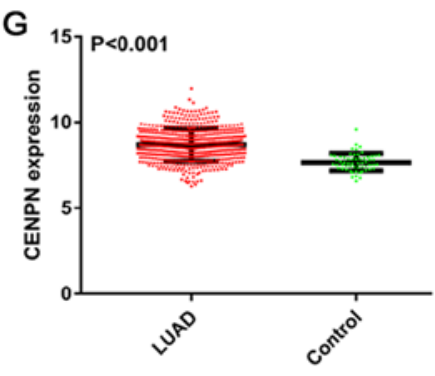

CENPN

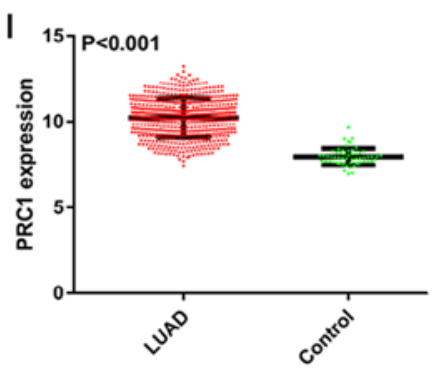

PRC1
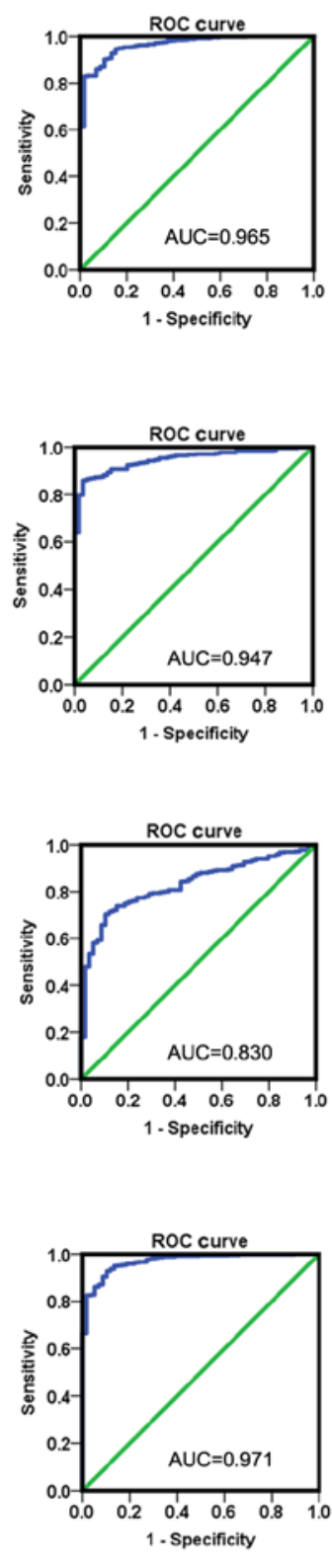

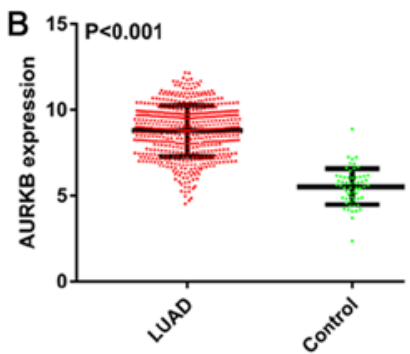

AURKB
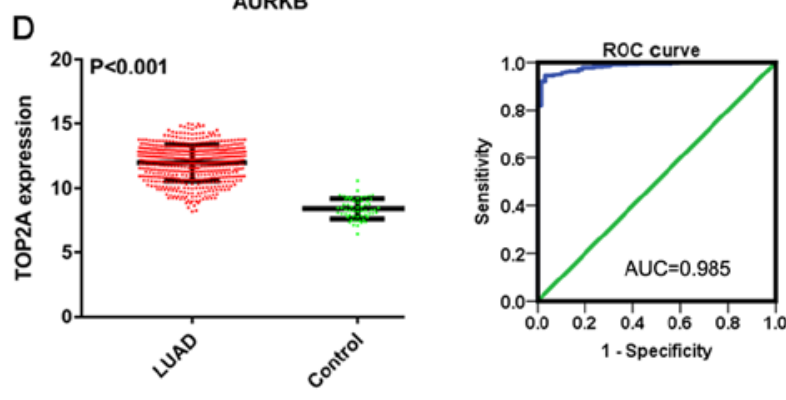

TOP2A

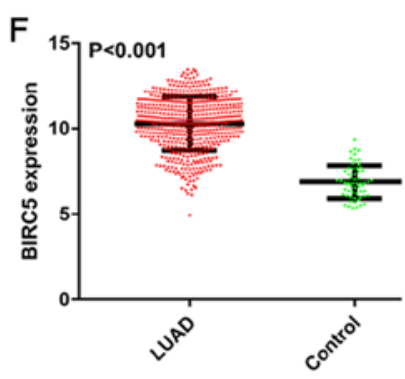

BIRC5
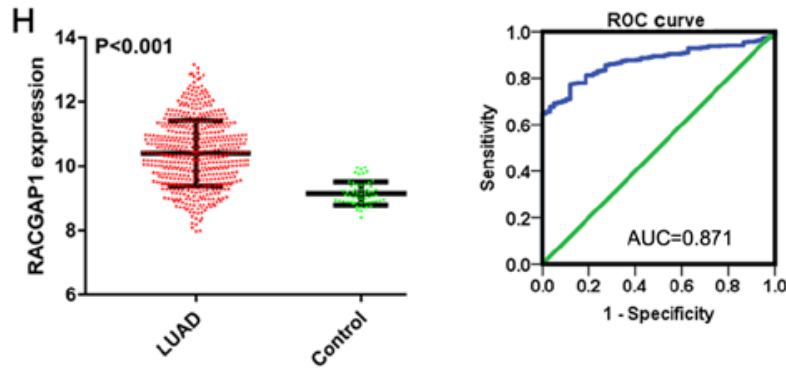

RACGAP1

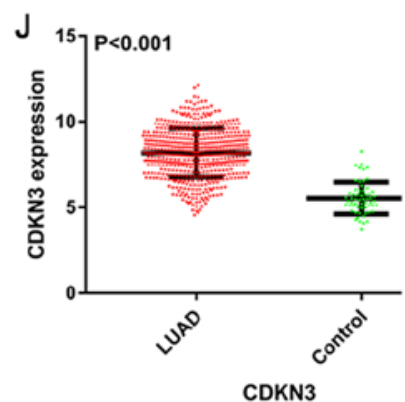

Figure 12. Scatter plots and ROC curves of 10 hub genes of microRNA-198-5p in LUAD based on data from The Cancer Genome Atlas. (A) CCNB2; (B) AURKB; (C) CCNA2; (D) TOP2A; (E) AURKA; (F) BIRC5; (G) CENPN; (H) RACGAP1; (I) PRC1; and (J) CDKN3. ROC, receiver operating characteristic; LUAD, lung adenocarcinoma, CCNB2, G2/mitotic-specific cyclin-B2; AURKB, Aurora kinase B; CCNA2, cyclin-A2; TOP2A, DNA topoisomerase 2- $\alpha$; AURKA, Aurora kinase A; BIRC5, baculoviral IAP repeat-containing protein 5; CENPN, centromere protein N; RACGAP1, Rac GTPase-activating protein 1; PRC1, protein regulator of cytokinesis 1; CDKN3, cyclin-dependent kinase inhibitor 3.

platinum drug resistance and NAFLD have not been previously reported in the context of miR-198-5p in LUAD. The p53 gene is a well-known tumor suppressor, and the p53 pathway is involved in several crucial processes of tumorigenesis, such as cell cycle arrest (53). A previous study revealed enhanced cell apoptosis and cell cycle arrests in LUAD cells by miR-198-5p, by targeting serine hydroxymethyltransferase 1 (54). This study did not investigate the involvement of the 
Table IV. KEGG pathway enrichment of the potential target genes of microRNA-198-5p in lung adenocarcinoma.

\begin{tabular}{|c|c|c|c|c|}
\hline Category & ID & Description & Count, $\mathrm{n}$ & P-value \\
\hline KEGG_PATHWAY & hsa04115 & p53 signaling pathway & 8 & $1.42 \times 10^{-6}$ \\
\hline KEGG_PATHWAY & hsa04110 & Cell cycle & 8 & $1.21 \times 10^{-4}$ \\
\hline KEGG_PATHWAY & hsa05012 & Parkinson's disease & 8 & $3.10 \times 10^{-4}$ \\
\hline KEGG_PATHWAY & hsa05010 & Alzheimer's disease & 8 & $1.07 \times 10^{-3}$ \\
\hline KEGG_PATHWAY & hsa00190 & Oxidative phosphorylation & 7 & $1.12 \times 10^{-3}$ \\
\hline KEGG_PATHWAY & hsa04914 & Progesterone-mediated oocyte maturation & 6 & $1.2 \times 10^{-3}$ \\
\hline KEGG_PATHWAY & hsa01524 & Platinum drug resistance & 5 & $1.86 \times 10^{-3}$ \\
\hline KEGG_PATHWAY & hsa04932 & Non-alcoholic fatty liver disease (NAFLD) & 7 & $2.15 \times 10^{-3}$ \\
\hline
\end{tabular}

KEGG; Kyoto Encyclopedia of Genes and Genomes.
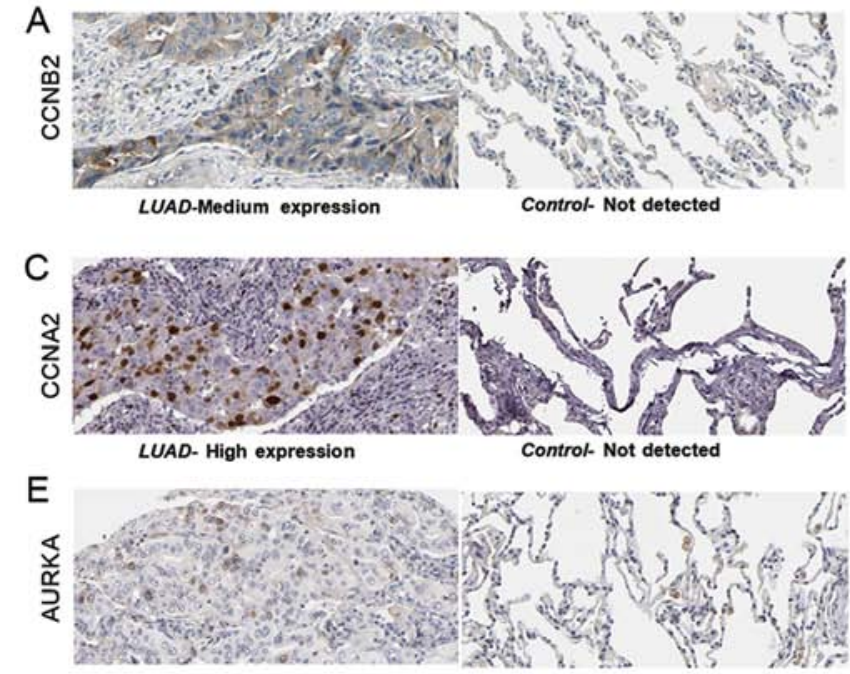

LUAD. Low expression

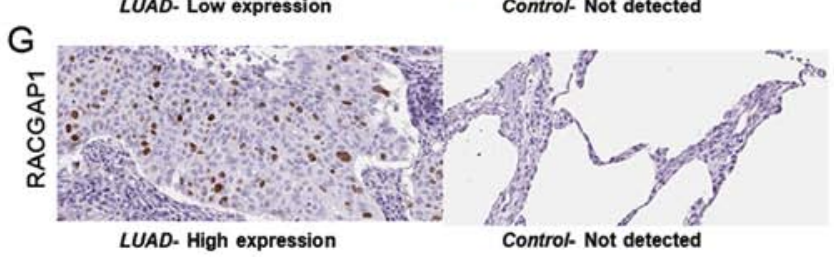

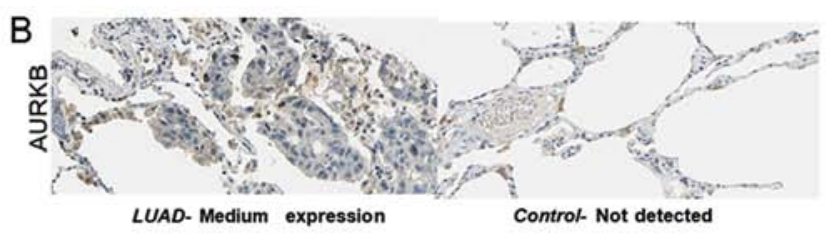
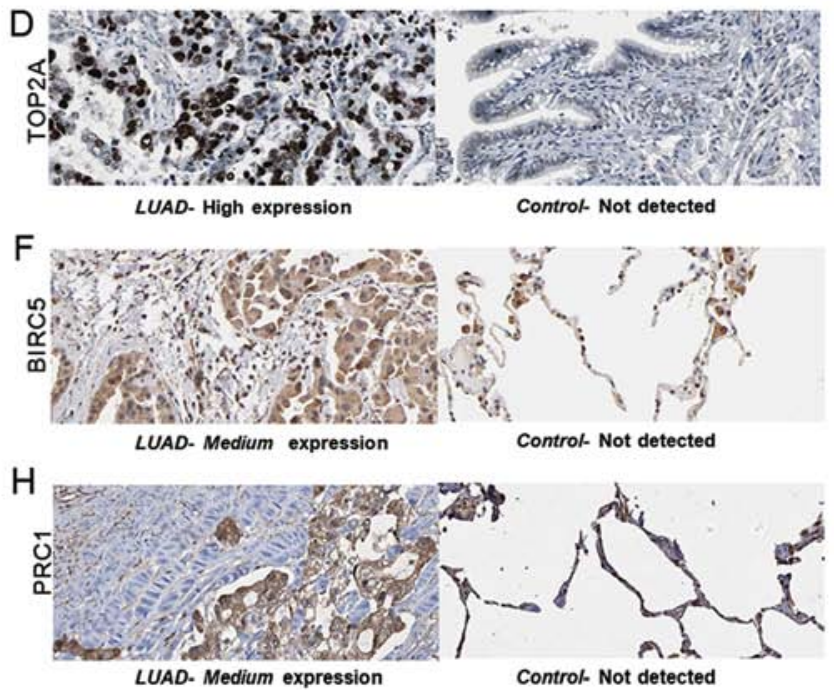

Figure 13. The expression pattern of the hub genes in LUAD tissues and adjacent normal lung tissues (control), obtained from The Human Protein Atlas database. (A) CCNB2 protein was moderately expressed in LUAD tissue. (B) Medium expression of AURKB was observed in LUAD tissue. (C) CCNA2 was highly expressed in LUAD tissue. (D) TOP2A was highly expressed in LUAD tissue. (E) AURKA expression was low in LUAD. (F) Medium expression of BIRC5 in LUAD tissue. (G) High expression of RACGAP1 in LUAD tissue. (H) PRC1 protein staining was medium in LUAD tissues. For all proteins, no staining was observed in normal lung tissue. LUAD, lung adenocarcinoma, CCNB2, G2/mitotic-specific cyclin-B2; AURKB, Aurora kinase B; CCNA2, cyclin-A2; TOP2A, DNA topoisomerase 2- $\alpha$; AURKA, Aurora kinase A; BIRC5, baculoviral IAP repeat-containing protein 5; RACGAP1, Rac GTPase-activating protein 1; PRC1, protein regulator of cytokinesis 1 .

p53 pathway. A lower miR-198-5p expression was speculated to be one of the candidate biomarkers for Parkinson's disease (36), which was substantiated by bioinformatics analysis to some extent. In the GO BP category, the putative targets participated mainly in mitotic nuclear division. For $\mathrm{CC}$, the target genes were enriched mainly in the mitochondrial respiratory chain complex IV. For MF, the target genes associated with cytochrome $c$ oxidase activity.

The potential targets of miR-198-5p were CCNB2, AURKB, CCNA2, TOP2A, AURKA, BIRC5, CENPN, RACGAP1, PRC1 and $\mathrm{CDKN} 3$, whose expression was significantly upregulated in LUAD samples (according to TCGA data). The upregulation of eight of these hub genes was subsequently validated at the protein level, where data from THPA showed positive staining in LUAD samples, whereas no staining was detected in non-tumor lung tissues. The overexpression of these hub genes could be ascribed to the dysfunction or downregulation of miR-198-5p. The miRNA-target gene network is quite complex; one mRNA may be regulated by disparate miRNAs, and a single miRNA molecule could target multiple genes in different diseases (55-57). Hence, identifying the exact mechanism of miR-198-5p and its target genes requires further investigation. The findings of the present study provide a computational biology perspective, rather than a focus on specific target genes. 
Several limitations of the present study should be noted. When distinguishing between LUAD and non-tumor tissues, the expression of miR-198-5p in bodily fluid samples was not clear, which limits its application in diagnosis. As mentioned, the treatment-associated information of the patients was incomplete, which weakened the reliability of the outcomes. Other experimental methods such as fluorescence in situ hybridization (FISH) were not used to verify the downregulation of miR-198-5p in LUAD samples, as RT-qPCR is regarded as the golden standard for miRNA quantification $(58,59)$ and the level of certain miRNAs in NSCLC detected by FISH was consistent with that detected by RT-Qpcr (60). Moreover, the findings of the present study are based mainly on online databases and in silico bioinformatics analyses. Based on TCGA and THPA, the hub genes of miR-198-5p were upregulated in LUAD both at the RNA level and the protein level; however, their upregulation in LUAD tissues remains to be further verified by quantitative means, such as RT-qPCR and western blotting. The role of miR-198-5p and its mechanism in LUAD should be investigated with further in vitro and in vivo experiments.

In conclusion, miR-198-5p is downregulated in LUAD tissues, and it functions as a prognostic factor. Based on the combined results of the in-house RT-qPCR, data mining and bioinformatics analysis, miR-198-5p was demonstrated to be involved in the development of LUAD by targeting various signaling pathways.

\section{Acknowledgements}

Not applicable.

\section{Funding}

The study was supported by the Youth Science Foundation of Guangxi Medical University, Nanning, China (grant no. GXMUYSF2014032), the Central Government Guide Local Science and Technology Development Project, China (grant no. ZY18076006), Fund of National Natural Science Foundation of China (grant no. NSFC81560469), Natural Science Foundation of Guangxi, China (grant no. 2017GXNSFAA198016), and Guangxi Degree and Postgraduate Education Reform and Development Research Projects, China (grant no.JGY2019050)

\section{Availability of data and materials}

All data generated or analyzed during this study are included in this published article.

\section{Authors' contributions}

YYF, SSW, LJP, and GC conceived and designed the study. YYF, SSW, JCH, YYL, and YNG collected, extracted and analyzed the data. YYF, JCH, and YNG drafted the manuscript. LJP and GC critically revised the manuscript. All authors read and approved the final manuscript.

\section{Ethics approval and consent to participate}

The Medical Ethics Committee of the First Affiliated Hospital of Guangxi Medical University (Nanning, China) approved the present study (approval no. 2015 KY-E-041). Each participant signed an informed consent form.

\section{Patient consent for publication}

Not applicable.

\section{Competing interests}

The authors declare that they have no competing interests.

\section{References}

1. Siegel RL, Miller KD and Jemal A: Cancer statistics, 2018. CA Cancer J Clin 68: 7-30, 2018.

2. Chen W, Zheng R, Baade PD, Zhang S, Zeng H, Bray F, Jemal A, Yu XQ and He J: Cancer statistics in China, 2015. CA Cancer J Clin 66: 115-132, 2016.

3. Siegel RL, Miller KD and Jemal A: Cancer statistics, 2017. CA Cancer J Clin 67: 7-30, 2017.

4. Siegel RL, Miller KD and Jemal A: Cancer statistics, 2016. CA Cancer J Clin 66: 7-30, 2016.

5. Ettinger DS: Ten years of progress in non-small cell lung cancer. J Natl Compr Canc Netw 10: 292-295, 2012.

6. Noone AM, Cronin KA, Altekruse SF, Howlader N, Lewis DR, Petkov VI and Penberthy L: Cancer incidence and survival trends by subtype using data from the surveillance epidemiology and end results program, 1992-2013. Cancer Epidemiol Biomarkers Prev 26: 632-641, 2017.

7. Berindan-Neagoe I, Monroig Pdel C, Pasculli B and Calin GA: MicroRNAome genome: A treasure for cancer diagnosis and therapy. CA Cancer J Clin 64: 311-336, 2014.

8. Yanaihara N, Caplen N, Bowman E, Seike M, Kumamoto K, Yi M, Stephens RM, Okamoto A, Yokota J, Tanaka T, et al: Unique microRNA molecular profiles in lung cancer diagnosis and prognosis. Cancer Cell 9: 189-198, 2006.

9. Lin Y, Lv Y, Liang R, Yuan C, Zhang J, He D, Zheng X and Zhang J: Four-miRNA signature as a prognostic tool for lung adenocarcinoma. Onco Targets Ther 11: 29-36, 2018.

10. Murakami T, Kikuchi H, Ishimatsu H, Iino I, Hirotsu A, Matsumoto T, Ozaki Y, Kawabata T, Hiramatsu Y, Ohta M, et al: Tenascin $\mathrm{C}$ in colorectal cancer stroma is a predictive marker for liver metastasis and is a potent target of miR-198 as identified by microRNA analysis. Br J Cancer 117: 1360-1370, 2017.

11. Li Y, Luo H, Xiao N, Duan J, Wang Z and Wang S: Long noncoding RNA SChLAP1 accelerates the proliferation and metastasis of prostate cancer via targeting miR-198 and promoting the MAPK1 pathway. Onco Res 26: 131-143, 2018.

12. Hu Y, Tang Z, Jiang B, Chen J and Fu Z: miR-198 functions as a tumor suppressor in breast cancer by targeting CUB domain-containing protein 1. Oncol Lett 13: 1753-1760, 2017.

13. Yang J, Zhao H, Xin Y and Fan L: MicroRNA-198 inhibits proliferation and induces apoptosis of lung cancer cells via targeting FGFR1. J Cell Biochem 115: 987-995, 2014.

14. Mirsadraee S, Oswal D, Alizadeh Y, Caulo A and van Beek E Jr: The 7th lung cancer TNM classification and staging system: Review of the changes and implications. World J Radiol 4: 128-134, 2012.

15. Peltier HJ and Latham GJ: Normalization of microRNA expression levels in quantitative RT-PCR assays: Identification of suitable reference RNA targets in normal and cancerous human solid tissues. RNA 14: 844-852, 2008.

16. Livak KJ and Schmittgen TD: Analysis of relative gene expression data using real-time quantitative PCR and the 2(-Delta Delta C(T)) method. Methods 25: 402-408, 2001.

17. Barrett T, Troup DB, Wilhite SE,Ledoux P, Rudnev D, Evangelista C, Kim IF, Soboleva A, Tomashevsky M and Edgar R: NCBI GEO: Mining tens of millions of expression profiles-database and tools update. Nucleic Acids Res 35: D760-D765, 2006.

18. Parkinson H, Kapushesky M, Shojatalab M, Abeygunawardena N, Coulson R, Farne A, Holloway E, Kolesnykov N, Lilja P, Lukk M, et al: ArrayExpress-a public database of microarray experiments and gene expression profiles. Nucleic Acids Res 35: D747-D750, 2007.

19. Han HS, Yun J, Lim SN, Han JH, Lee KH, Kim ST, Kang MH, Son SM, Lee YM, Choi SY, et al: Downregulation of cell-free miR-198 as a diagnostic biomarker for lung adenocarcinoma-associated malignant pleural effusion. Int J Cancer 133: 645-652, 2013. 
20. Anders S and Huber W: Differential expression analysis for sequence count data. Genome Biol 11: R106, 2010.

21. Dweep H and Gretz N: miRWalk2.0: A comprehensive atlas of microRNA-target interactions. Nat Methods 12: 697, 2015.

22. Ashburner M, Ball CA, Blake JA, Botstein D, Butler H, Cherry JM, Davis AP, Dolinski K, Dwight SS, Eppig JT, et al: Gene Ontology: Tool for the unification of biology. The Gene Ontology Consortium. Nat Genet 25: 25-29, 2000.

23. Kanehisa M and Goto S: KEGG: Kyoto encyclopedia of genes and genomes. Nucleic Acids Res 28: 27-30, 2000.

24. Dennis G Jr, Sherman BT, Hosack DA, Yang J, Gao W, Lane HC and Lempicki RA: DAVID: Database for annotation, visualization, and integrated discovery. Genome Biol 4: P3, 2003.

25. Yu G, Wang LG, Han Y and He QY: clusterProfiler: An R package for comparing biological themes among gene clusters. OMICS 16: 284-287, 2012.

26. Higgins JP, Thompson SG, Deeks JJ and Altman DG: Measuring inconsistency in meta-analyses. BMJ 327: 557-560, 2003.

27. Seike M, Goto A, Okano T, Bowman ED, Schetter AJ, Horikawa I, Mathe EA, Jen J, Yang P, Sugimura H, et al: MiR-21 is an EGFR-regulated anti-apoptotic factor in lung cancer in never-smokers. Proc Natl Acad Sci USA 106: 12085-12090, 2009.

28. Nymark P, Guled M, Borze I, Faisal A, Lahti L, Salmenkivi K, Kettunen E, Anttila S and Knuutila S: Integrative analysis of microRNA, mRNA and aCGH data reveals asbestos- and histology-related changes in lung cancer. Genes Chromosomes Cancer 50: 585-597, 2011

29. Bjaanaes MM, Halvorsen AR, Solberg S, Jørgensen L, Dragani TA, Galvan A, Colombo F, Anderlini M, Pastorino U, Kure E, et al: Unique microRNA-profiles in EGFR-mutated lung adenocarcinomas. Int J Cancer 135: 1812-1821, 2014.

30. Tai MC, Kajino T, Nakatochi M, Arima C, Shimada Y, Suzuki M, Miyoshi H, Yatabe Y, Yanagisawa K and Takahashi T: miR-342-3p regulates MYC transcriptional activity via direct repression of E2F1 in human lung cancer. Carcinogenesis 36: 1464-1473, 2015

31. Robles AI, Arai E, Mathé EA, Okayama H, Schetter AJ, Brown D, Petersen D, Bowman ED, Noro R, Welsh JA, et al: An integrated prognostic classifier for stage I lung adenocarcinoma based on mRNA, microRNA, and DNA methylation biomarkers. J Thorac Oncol 10: 1037-1048, 2015.

32. Lu J, Kwan BC, Lai FM, Tam LS, Li EK, Chow KM, Wang G, Li PK and Szeto CC: Glomerular and tubulointerstitial miR-638, miR-198 and miR-146a expression in lupus nephritis. Nephrology (Carlton) 17: 346-351, 2012

33. Zhang Z, Chang H, Li Y, Zhang T, Zou J, Zheng X and Wu J: MicroRNAs: Potential regulators involved in human anencephaly. Int J Biochem Cell Biol 42: 367-374, 2010.

34. Bakre A, Mitchell P, Coleman JK, Jones LP, Saavedra G Teng M, Tompkins SM and Tripp RA: Respiratory syncytial virus modifies microRNAs regulating host genes that affect virus replication. J Gen Virol 93: 2346-2356, 2012.

35. Choi SY, Yun J, Lee OJ, Han HS, Yeo MK, Lee MA and Suh KS: MicroRNA expression profiles in placenta with severe preeclampsia using a PNA-based microarray. Placenta 34 799-804, 2013.

36. Cardo LF, Coto E, Ribacoba R, Menéndez M, Moris G, Suárez E and Alvarez V: MiRNA profile in the substantia nigra of Parkinson's disease and healthy subjects. J Mol Neurosci 54: 830-836, 2014

37. Qi B, Yao WJ, Zhao BS, Qin XG, Wang Y, Wang WJ, Wang TY, Liu SG and Li HC: Involvement of microRNA-198 overexpression in the poor prognosis of esophageal cancer. Asian Pac J Cancer Prev 14: 5073-5076, 2013.

38. Bi C, Chung TH, Huang G, Zhou J, Yan J, Ahmann GJ, Fonseca R and Chng WJ: Genome-wide pharmacologic unmasking identifies tumor suppressive microRNAs in multiple myeloma. Oncotarget 6: 26508-26518, 2015.

39. Vychytilova-Faltejskova P,Kiss I, Klusova S,Hlavsa J,Prochazka V, Kala Z, Mazanec J, Hausnerova J, Kren L, Hermanova M, et al: MiR-21, miR-34a, miR-198 and miR-217 as diagnostic and prognostic biomarkers for chronic pancreatitis and pancreatic ductal adenocarcinoma. Diagn Pathol 10: 38, 2015.

40. Zhao JJ, Yang J, Lin J, Yao N, Zhu Y, Zheng J, Xu J, Cheng JQ, Lin JY and MA J: Identification of miRNAs associated with tumorigenesis of retinoblastoma by miRNA microarray analysis. Childs Nerv Syst 25: 13-20, 2009.

41. Wong TS, Liu XB, Wong BY, Ng RW, Yuen AP and Wei WI: Mature miR-184 as potential oncogenic microRNA of squamous cell carcinoma of the tongue. Clin Cancer Res 14: 2588-2592, 2008
42. Zhang S, Zhao Y and Wang L: MicroRNA-198 inhibited tumorous behaviors of human osteosarcoma through directly targeting ROCK1. Biochem Biophys Res Commun 472: 557-565, 2016.

43. Nie E, Jin X, Wu W, Yu T, Zhou X, Shi Z, Zhang J, Liu N and You Y: MiR-198 enhances temozolomide sensitivity in glioblastoma by targeting MGMT. J Neurooncol 133: 59-68, 2017.

44. Wang M, Wang J, Kong X, Chen H, Wang Y, Qin M, Lin Y, Chen $\mathrm{H}, \mathrm{Xu} \mathrm{J}$, Hong J, et al: MiR-198 represses tumor growth and metastasis in colorectal cancer by targeting fucosyl transferase 8 . Sci Rep 4: 6145, 2014

45. Cui Z, Zheng X and Kong D: Decreased miR-198 expression and its prognostic significance in human gastric cancer. World J Surg Oncol 14: 33, 2016

46. Marin-Muller C, Li D, Bharadwaj U, Li M, Chen C, Hodges SE, Fisher WE, Mo Q, Hung MC and Yao Q: A tumorigenic factor interactome connected through tumor suppressor microRNA-198 in human pancreatic cancer. Clin Cancer Res 19: 5901-5913, 2013.

47. Huang WT, Wang HL, Yang H, Ren FH, Luo YH, Huang CQ, Liang YY, Liang HW, Chen G and Dang YW: Lower expressed miR-198 and its potential targets in hepatocellular carcinoma: A clinicopathological and in silico study. Onco Targets Ther 9: 5163-5180, 2016

48. Gigante M, Pontrelli P, Herr W, Gigante M, D'Avenia M, Zaza G, Cavalcanti E, Accetturo M, Lucarelli G, Carrieri G, et al: miR-29b and miR-198 overexpression in CD8 ${ }^{+} \mathrm{T}$ cells of renal cell carcinoma patients down-modulates JAK3 and MCL-1 leading to immune dysfunction. J Transl Med 14: 84, 2016.

49. Liang YY, Huang JC, Tang RX, Chen WJ, Chen P, Cen WL, Shi K, Gao L, Gao X, Liu AG, et al: Clinical value of miR-198-5p in lung squamous cell carcinoma assessed using microarray and RT-qPCR. World J Surg Oncol 16: 22, 2018.

50. Han HS, Jo YN, Lee JY, Choi SY, Jeong Y, Yun J and Lee OJ: Identification of suitable reference genes for the relative quantification of microRNAs in pleural effusion. Oncol Lett 8 1889-1895, 2014

51. Ettinger DS, Wood DE, Aisner DL, Akerley W, Bauman J, Chirieac LR, D'Amico TA, DeCamp MM, Dilling TJ, Dobelbower M, et al: Non-small cell lung cancer, version 5.2017, NCCN clinical practice guidelines in oncology. J Natl Compr Canc Netw 15: 504-535, 2017.

52. Zagryazhskaya A and Zhivotovsky B: miRNAs in lung cancer: A link to aging. Ageing Res Rev 17: 54-67, 2014.

53. Ozaki T and Nakagawara A: Role of $\mathrm{p} 53$ in cell death and human cancers. Cancers (Basel) 3: 994-1013, 2011.

54. Wu S, Zhang G, Li P, Chen S, Zhang F, Li J, Jiang C, Chen X, Wang Y, Du Y, et al: miR-198 targets SHMT1 to inhibit cell proliferation and enhance cell apoptosis in lung adenocarcinoma. Tumour Biol 37: 5193-5202, 2016.

55. Hausser $\mathbf{J}$ and Zavolan $\mathrm{M}$ : Identification and consequences of miRNA-target interactions-beyond repression of gene expression. Nat Rev Genet 15: 599-612, 2014.

56. Nakanishi K: Anatomy of RISC: How do small RNAs and chaperones activate Argonaute proteins? Wiley Interdiscip Rev RNA 7: 637-660, 2016.

57. Beermann J, Piccoli MT, Viereck J and Thum T: Non-coding RNAs in development and disease: Background, mechanisms, and therapeutic approaches. Physiol Rev 96: 1297-325, 2016.

58. Duan D, Zheng KX, Shen Y, Cao R, Jiang L, Lu Z, Yan X and $\mathrm{Li}$ J: Label-free high-throughput microRNA expression profiling from total RNA. Nucleic Acids Res 39: e154, 2011.

59. Ouyang T, Liu Z, Han Z and Ge Q: MicroRNA detection specificity: Recent advances and future perspective. Anal Chem 91 3179-3186, 2019.

60. Li J, Yang H, Li Y, Liu Y, Chen S, Qi C, Zhang Q, Lan T, He X, Guan XY and Wang L: microRNA-146 up-regulation predicts the prognosis of non-small cell lung cancer by miRNA in situ hybridization. Exp Mol Pathol 96: 195-199, 2014

61. Travis WD, Brambilla E, Noguchi M, Nicholson AG, Geisinger KR, Yatabe Y, Beer DG, Powell CA, Riely GJ, Van Schil PE, et al: International association for the study of lung cancer/americanthoracic society/european respiratory society internationalmultidisciplinary classification of lung adenocarcinoma. J Thorac Oncol 6: 244-285, 2011.

This work is licensed under a Creative Commons Attribution-NonCommercial-NoDerivatives 4.0 International (CC BY-NC-ND 4.0) License. 